\title{
HEAT KERNEL ESTIMATES AND FUNCTIONAL CALCULI OF $-b \Delta$
}

\author{
ALAN MCINTOSH and ANDREA NAHMOD
}

\begin{abstract}
We show that the elliptic operator $\mathscr{L}=-b(x) \Delta$ has a bounded $H^{\infty}$ functional calculus in $L^{p}\left(\mathrm{R}^{n}\right), 1<p<\infty$, where $b$ is a bounded measurable complex-valued function with positive real part. In the process, we prove quadratic estimates for $\mathscr{L}$, and obtain bounds with fast decay and Hölder continuity estimates for $k_{t}(x, y) b(y)$ and its gradient, where $k_{t}(x, y)$ is the heat kernel of $-b(x) \Delta$. This implies $L^{p}$ regularity of solutions to the parabolic equation $\partial_{t} u+\mathscr{L} u=0$.
\end{abstract}

\section{Introduction}

We consider the operator $\mathscr{L}=-b(x) \Delta$, a special case of a second order elliptic operator in non-divergence form with bounded measurable complex coefficients. Here $b \in L^{\infty}\left(\mathbf{R}^{n}, \mathbf{C}\right)$, and $\operatorname{Re} b(x) \geq \kappa$ for some $\kappa>0$ and almost all $x \in \mathrm{R}^{n}$. Our main aim is to prove that $\mathscr{L}$ has a bounded $H^{\infty}$ functional calculus in $L^{p}\left(\mathrm{R}^{n}\right), 1<p<\infty$, meaning that $\|f(-b \Delta)\| \leq c_{p}\|f\|_{\infty}$ for all bounded holomorphic functions defined on a suitable sector in the complex plane.

In the course of the proof, we show that the kernel of $e^{-t \mathscr{L}} b$ is a $C^{1, \eta}\left(\mathrm{R}^{n} \times \mathrm{R}^{n}\right)$ function for all $\eta<1$, with bounds, and Hölder bounds on its gradient, decaying polynomially fast. In particular, this means that the heat kernel, namely the kernel of $e^{-t \mathscr{L}}$, along with its derivative with respect to $x$, satisfies Holder estimates in the $x$-variable with polynomially decaying bounds. This implies $L^{p}$ regularity of solutions to the parabolic equation $\partial_{t} u+\mathscr{L} u=0$. Regularity in the $y$-variable holds for the kernel of $e^{-t \mathscr{L}} b$.

The strategy used to prove the results is this. First we prove that for $k$ large enough, the kernel of $(\mathscr{L}-\lambda I)^{-k} b$, the iterated resolvent of $-b \Delta$ times $b$, is a $C^{1, \eta}\left(\mathrm{R}^{n} \times \mathrm{R}^{n}\right)$ function. Next by means of commutators, we prove that the bounds and Hölder bounds of this kernel and its gradient have polynomial decay away from the diagonal. By means of an appropriate contour

The authors received support from the Australian Government through the ARC.

Received December 11, 1997. 
integral, the bounds and Hölder continuity of the kernel of $e^{-t \mathscr{L}} b$ and its gradient readily follow, as do bounds on the kernel of $(\mathscr{L}-\lambda I)^{-1} b$.

For the functional calculus, we first prove that $\mathscr{L}$ has a bounded $H^{\infty}$ functional calculus in $L^{2}\left(\mathrm{R}^{n}\right)$. This is equivalent to proving that $\mathscr{L}$ and its dual $\mathscr{L}^{\prime}$ satisfy quadratic estimates [30]. The quadratic estimates are obtained by using the above-mentioned kernel bounds, together with a result of Semmes [36].

These kernel bounds can also be employed to show that the kernel of $f(\mathscr{L}) b$, where $f$ is a bounded holomorphic function on a suitable sector, satisfies Calderón-Zygmund estimates. Thus $\mathscr{L}$ has a bounded $H^{\infty}$ functional calculus in $L^{p}\left(\mathrm{R}^{n}\right)$ for $1<p<\infty$.

In the final section we present a range of extensions and applications of these results. (i) We prove that the generalized Riesz transforms $\frac{\partial}{\partial x_{j}} \mathscr{L}^{-\frac{1}{2}}$ are bounded operators on $L^{p}\left(\mathrm{R}^{n}\right)$ for $1<p<\infty$. (ii) We give a positive answer to the square root problem for $\Delta b \Delta$. (iii) We mention that the boundedness of the functional calculus in $L^{p}\left(\mathrm{R}^{n}\right)$ implies maximal regularity results for the parabolic equation $\frac{\partial u}{\partial t}(t)+\mathscr{L} u(t)=f(t), u(0)=0$ for $t>0$ [21]. (iv) We remark that the polynomial decay of the various kernels can be improved to exponential decay. (v) We note that the results remain true when $b$ is replaced by an invertible bounded matrix-valued $\omega$-accretive function $B$ and $L^{p}\left(\mathrm{R}^{n}\right)$ is replaced by $L^{p}\left(\mathrm{R}^{n}, \mathrm{C}^{m}\right)$. (vi) We note that $b(-\Delta)^{r}$ has a bounded $H^{\infty}$ functional calculus in $L^{p}\left(\mathrm{R}^{n}\right), 1<p<\infty$, for $r$ large enough. This is obtained by proving that the kernel of $\left(b(-\Delta)^{r}-\lambda I\right)^{-2} b$ and its gradient are bounded with sufficient decay away from the diagonal and satisfy Hölder estimates. (vii) We note that the operators continue to have bounded $H^{\infty}$ functional calculi when lower order terms are added. (viii) We show that the operator $b\left(-\Delta_{\Omega}+I\right)$ has a bounded $H^{\infty}$ functional calculus in $L^{2}(\Omega)$ when $\Delta_{\Omega}$ is the Laplacian subject to Dirichlet or Neumann boundary conditions on a bounded Lipschitz domain.

Results on holomorphic functional calculi in $L^{p}\left(\mathrm{R}^{n}\right)$ for second order elliptic operators in non-divergence form were obtained by David and Journé [16] for small $L^{\infty}$ perturbations of $-\Delta$. They relied on multilinear expansions and their T(1)-theorem. On the other hand Amann, Hieber and Simonett obtained bounded $H^{\infty}$ functional calculi in $L^{p}\left(\mathrm{R}^{n}\right)$ for higher order elliptic systems in non-divergence form with Hölder coefficients [3]. Recently Duong and Simonett have generalized both these results to the case of higher order elliptic systems whose coefficients are either small $L^{\infty}$ perturbations of constants, or are bounded and uniformly continuous [25]. They also relied on multilinear expansions, the $T(1)$-theorem of David and Journé, and a localization argument of Amann, Hieber and Simonett. How- 
ever their result does not include ours, because our function $b$ is not necessarily continuous nor a small perturbation of a constant.

Concerning the higher order operators, we refer to the work of Beals on the Green's kernel of self-adjoint elliptic operators of high enough order with minimally smooth coefficients [10]. Also see the book of Robinson [35]. More recently, the spectral theory of higher order elliptic operators with measurable coefficients was studied by Davies [19].

We study this operator $-b \Delta$ as a step towards a better understanding of functional calculi of elliptic operators in non-divergence form. Bear in mind however that Bauman has presented an example of a second order uniformly elliptic operator $\mathscr{L}$ in non-divergence form with continuous coefficients whose Green's function $g(x, y)$ is not locally bounded away from the diagonal $[8,9]$. This is achieved by proving some estimates on suitably "normalized" solutions to the adjoint equation $\mathscr{L}^{*} v=0$ and it demonstrates a lack of regularity for the solutions $v$ to the adjoint equation. (We avoid this problem for $-b \Delta$ by multiplying the heat kernel by $b(y)$.) On the other hand, for second order elliptic operators $\mathscr{L}$ in non-divergence form with real measurable coefficients, Krylov and Safonov [29] have obtained versions of the Harnack inequality for solutions to the parabolic equation (with time dependent coefficients) and derived from them the Holder continuity of the solutions. Furthermore, for such an $\mathscr{L}$, Fabes and Stroock [26] have established "backwards" Hölder inequalities for nonnegative solutions to $\mathscr{L}^{*} v=0$ and used them to obtain certain integrability properties for the heat kernel.

We remark that, for the operator $\mathscr{L}=-b \Delta$ with $b$ a smooth complexvalued function, regularity results for solutions to the parabolic equation associated with $\mathscr{L}$ are implicit in the work of Showalter [37], where existence and regularity of solutions to the partial differential equation of Sobolev type are studied.

The results in this paper are in the spirit of those in [7] and in [4] but differ significantly from those in two ways. In this paper no smoothness is assumed on $b$ and the only "regularity" result we rely on is the $L^{p}$-contractibility of the Bessel potentials. This is possible because of the specific form of the operator we study.

The $L^{2}$ results in this paper are essentially a generalization to higher dimensions of the $L^{2}$ boundedness of the Cauchy integral $C_{\gamma}$ on the Lipschitz graph $\gamma[12,13]$. This is because, in one dimension, $-b \Delta$ has a bounded $H^{\infty}$ functional calculus if and only if $-i \frac{d}{d z}=-i b \frac{d}{d x}$ does, where $\gamma$ is parametrized by $z=g(x)$ with $g^{\prime}(x)=\frac{1}{b}$. This in turn implies $C_{\gamma}: L^{2}(\gamma) \rightarrow L^{2}(\gamma)$ because $C_{\gamma}=\operatorname{sgn}\left(-i \frac{d}{d z}\right)$, where $\operatorname{sgn}$ is the $H^{\infty}\left(S_{\mu}^{0}\right)$ function defined by $\operatorname{sgn}(\zeta)= \pm 1$ when $|\arg ( \pm \zeta)|<\mu$. See, e.g. [1]. 
Acknowledgments. We wish to express our appreciation to Pascal Auscher, Xuan Duong and Carlos Kenig for contributing to our understanding of this material and for informing us about some of the above background material. We thank the International Centre for Mathematical Sciences in Edinburgh where our first lectures on this topic were presented in June 1994. The subsequent research was undertaken at Macquarie University in Sydney and the University of Texas at Austin.

\section{Notation and Terminology}

The integer $n$ always denotes the dimension. For $\alpha=\left(\alpha_{1}, \alpha_{2}, \ldots, \alpha_{n}\right) \in$ $\mathrm{Z}_{+}^{n}$, the operator $D^{\alpha}$ denotes differentiation of order $|\alpha|=\alpha_{1}+\alpha_{2}+\ldots \alpha_{n}$ in $\mathrm{R}^{n}$ and $\Delta=\sum_{j=1}^{n} \frac{\partial^{2}}{\partial x^{2}}$ denotes the Laplacian in $\mathrm{R}^{n}$.

For $1 \leq p \leq \infty, L^{p}=L^{p}\left(\mathrm{R}^{n}\right)$ is used for the Lebesgue space on $\mathrm{R}^{n}$; and $W^{k, p}=W^{k, p}\left(\mathrm{R}^{n}\right)$, with $k$ a positive integer, for the inhomogeneous Sobolev space of all functions $f$ on $\mathrm{R}^{n}$ for which $D^{\alpha} f$ is in $L^{p}$ for all $0 \leq|\alpha| \leq k$ (see [38] for example).

For $0 \leq \eta<1, \dot{C}^{\eta}$ denotes the space of functions on $\mathrm{R}^{n}$ which are uniformly Hölder continuous of order $\eta$, and $C^{\eta}=\dot{C}^{\eta} \cap L^{\infty}$. For any nonnegative integer $r, C^{r}$ denotes the Banach space consisting of all functions $f$ on $\mathbf{R}^{n}$ which together with all their derivatives $D^{\alpha} f$ of orders $|\alpha| \leq r$ are bounded and continuous on $\mathrm{R}^{n}$. The space of functions in $C^{r}$ which are also compactly supported is denoted by $C_{0}^{r}$. Finally, if $0<\eta \leq 1$ and $r$ is a nonnegative integer, $C^{r, \eta}$ denotes the subspace of $C^{r}$ consisting of those functions $f$ on $\mathrm{R}^{n}$ for which $D^{\alpha} f$ is Hölder continuous of order $\eta$ on $\mathrm{R}^{n}$ for all $0 \leq|\alpha| \leq r$.

For any two Banach spaces $B_{1}$ and $B_{2}$, we use $T: B_{1} \rightarrow B_{2}$ to express the fact that $T$ is a bounded linear operator from $B_{1}$ to $B_{2}$ and $\|\cdot\|_{B_{1}, B_{2}}$ to indicate its operator norm. When $B_{1}=L^{p}$ and $B_{2}=L^{q}$ we abbreviate $\|\cdot\|_{L^{p}, L^{q}}$ to $\|\cdot\|_{p, q}$.

Throughout this paper $0 \leq \omega<\frac{\pi}{2}$, and $\mathscr{L}=-b \Delta$ where $b$ denotes a bounded $\omega$-accretive function on $\mathrm{R}^{n}$ with bounded reciprocal, meaning that $b$ and $\frac{1}{b} \in L^{\infty}\left(\mathbf{R}^{n}, \mathrm{C}\right)$ and $|\arg b(x)| \leq \omega$ for almost all $x \in \mathbf{R}^{n}$. The domain of $\mathscr{L}$ in $L^{p}$ is $\mathscr{D}(\mathscr{L})=W^{2, p}\left(\mathrm{R}^{n}\right)$. When $p=2$, we let $\mathscr{L}^{\prime}$ denote the dual of $\mathscr{L}$ with respect to the pairing $\langle u, v\rangle=\int_{\mathrm{R}^{n}} u(x) v(x) d x$ between $L^{2}\left(\mathrm{R}^{n}\right)$ and itself. It is given by $\mathscr{L}^{\prime}=-\Delta b$ with $\mathscr{D}\left(\mathscr{L}^{\prime}\right)=\left\{v \in L^{2}\left(\mathrm{R}^{n}\right): b v \in W^{2,2}\left(\mathrm{R}^{n}\right)\right\}$.

\section{$\S 1$. Preliminary results}

For $0 \leq \omega<\mu<\pi$, define the closed and open sectors in the (extended) complex plane:

$$
S_{\omega+}=\{\zeta \in \mathrm{C}:|\arg \zeta| \leq \omega\} \cup\{0, \infty\}, \quad S_{\mu+}^{0}=\{\zeta \in \mathrm{C}: \zeta \neq 0,|\arg \zeta|<\mu\} .
$$


Denote by $H\left(S_{\mu+}^{0}\right)$ the space of all holomorphic functions on $S_{\mu+}^{0}$ and by $H^{\infty}\left(S_{\mu+}^{0}\right)=\left\{f \in H\left(S_{\mu_{+}}^{0}\right):\|f\|_{\infty}<\infty\right\}$ where $\|f\|_{\infty}=\sup \left\{|f(\zeta)|: \zeta \in S_{\mu_{+}}^{0}\right\}$. Let $\Psi\left(S_{\mu+}^{0}\right)=\psi \in H^{\infty}\left(S_{\mu+}^{0}\right):|\psi(\zeta)| \leq C|\zeta|^{s}\left(1+|\zeta|^{2 s}\right)^{-1}$ for some $\left.C, s>0\right\}$.

A closed operator $T$ in a Banach space $\mathscr{X}$ is said to be of type $\omega$ if its spectrum $\sigma(T) \subset S_{\omega+}$ and for each $\mu>\omega$ there exists $C_{\mu}$ such that

$$
\left\|(T-\zeta I)^{-1}\right\| \leq C_{\mu}|\zeta|^{-1}, \quad \zeta \notin S_{\mu+} .
$$

Every one-one operator with dense domain and dense range in a Banach space $\mathscr{X}$ has a unique holomorphic functional calculus which is consistent with the usual definition of polynomials of an operator [30, 1]. We remark that in a reflexive Banach space, every one-one operator $T$ of type $\omega$ necessarily has dense domain and dense range [15].

In particular, if $\psi \in \Psi\left(S_{\mu+}^{0}\right)$ then $\psi(T)=\frac{1}{2 \pi i} \int_{\Gamma}(T-\zeta I)^{-1} \psi(\zeta) d \zeta: \mathscr{H} \rightarrow \mathscr{H}$ where the integral is on the unbounded contour $\Gamma=\left\{\zeta=r e^{ \pm i \theta}: r \geq 0\right\}$, parametrized clockwise around $S_{\omega+}$, and $\omega<\theta<\mu$.

The question is whether, for all $f \in H^{\infty}\left(S_{\mu+}^{0}\right), f(T): \mathscr{X} \rightarrow \mathscr{X}$ with $\|f(T)\| \leq c_{\mu}|f| \|_{\infty}$. When this holds, we say that $T$ has a bounded $H^{\infty}\left(S_{\mu+}^{0}\right)$ functional calculus.

In [30] it was proved that, in the case when $\mathscr{X}$ is a Hilbert space $\mathscr{H}, T$ has a bounded $H^{\infty}$ functional calculus in $\mathscr{H}$ if and only $T$ and $T^{*}$ satisfy the quadratic estimates $\|u\|_{T}=\left\{\int_{0}^{\infty}\left\|\psi_{t}(T) u\right\|^{2} \frac{d t}{t}\right\}^{\frac{1}{2}} \leq c\|u\|$ and $\|u\|_{T^{*}} \leq c\|u\|$ where $\psi_{t}(\zeta)=\psi(t \zeta)$ and $\psi$ is any non-zero function in $\Psi\left(S_{\mu+}^{0}\right)$. Note that different choices of $\mu>\omega$ and of $\psi \in \Psi\left(S_{\mu+}^{0}\right)$ lead to equivalent quadratic norms $\|u\|_{T}[32,1]$.

The adjoint operator $T^{*}$ can be replaced by a dual operator $T^{\prime}$ with respect to any dual pairing of $\mathscr{H}$ with another Hilbert space. In this paper we use the dual with respect to the pairing $\langle u, v\rangle=\int_{\mathrm{R}^{n}} u(x) v(x) d x$ between $\mathscr{H}=L^{2}\left(\mathrm{R}^{n}\right)$ and itself.

Let us call $B$ a bounded $\omega$-accretive operator on a Hilbert space $\mathscr{H}$ if $B: \mathscr{H} \rightarrow \mathscr{H}$ and $|\arg (B u, u)| \leq \omega$ for all $u \in \mathscr{H}$. For such an operator, the spectrum $\sigma(B) \subset S_{\omega+}$. In particular, the operator of multiplication by the function $b$ is an invertible bounded $\omega$-accretive operator on $L^{2}\left(\mathrm{R}^{n}\right)$, meaning that in addition it has a bounded inverse.

Any operator $T$ of the form $A S$ or $S A$, where $S$ is a positive self-adjoint operator in $\mathscr{H}$, and $A$ is an invertible bounded $\omega$-accretive operator on $\mathscr{H}$, is a one-one operator of type $\omega$ in $\mathscr{H}[30,5]$. In particular this implies part (1) of Proposition 1.1 below. 
Proposition 1.1.

(1) The operators $\mathscr{L}$ and $\mathscr{L}^{\prime}$ are one-one operators of type $\omega$ in $L^{2}\left(\mathrm{R}^{n}\right)$;

(2) $\mathscr{L}$ is of type $\pi / 2+\omega$ in $L^{p}\left(\mathrm{R}^{n}\right)$ when $1 \leq p \leq \infty$. It is one-one when $1 \leq p<\infty$.

Proof. (2) Let $\mu>\omega$ be given. For each $\lambda \notin S_{(\pi / 2+\mu)+}$ there exists $\rho>0$ and a function $a \in L^{\infty}\left(\mathrm{R}^{n}\right)$ with $\|a\|_{\infty}<1$ such that $\frac{\lambda}{b}=\rho|\lambda|(-1+a)$. Then

$$
\begin{aligned}
(\mathscr{L}-\lambda I)^{-1} & =\left(-\Delta-\frac{\lambda}{b}\right)^{-1} \frac{1}{b}=(-\Delta+\rho|\lambda|-\rho|\lambda| a)^{-1} \frac{1}{b} \\
& =(-\Delta+\rho|\lambda|)^{-1} \frac{1}{b}+(-\Delta+\rho|\lambda|)^{-1} \rho|\lambda| a(-\Delta+\rho|\lambda|)^{-1} \frac{1}{b}+\ldots
\end{aligned}
$$

Now $\left\|\left(-\Delta+\alpha^{-2} I\right)^{-1}\right\|_{p, p} \leq \alpha^{2}$ for all $\alpha>0$, because $\left(-\alpha^{2} \Delta+I\right)^{-1}$ has kernel $\frac{1}{\alpha^{n}} G_{2}\left(\frac{x-y}{\alpha}\right)$ where $G_{2}$ denotes the Bessel potential which has $L^{1}$ norm equal to 1 [38, pp. 130-138]. On applying this fact with $\alpha^{-2}=\rho|\lambda|$ we obtain

$$
\left\|(\mathscr{L}-\lambda I)^{-1}\right\|_{p, p} \leq \frac{1}{\rho|\lambda|}\left(1+\|a\|_{\infty}+\|a\|_{\infty}^{2}+\ldots\right)\left\|\frac{1}{b}\right\|_{\infty} \leq \frac{c_{\mu}}{|\lambda|} .
$$

That $\mathscr{L}$ is one-one we leave to the reader.

REMARK. In Theorem 3.3 we shall use kernel bounds to show that $\mathscr{L}$ is actually of type $\omega$ in $L^{p}\left(\mathrm{R}^{n}\right), 1 \leq p \leq \infty$. Part (1) below is also improved in Corollary 3.4 .

Proposition 1.2. Let $\mu>\omega$. For any $\lambda \notin S_{(\pi / 2+\mu)+},|\lambda|=1$ we have that

(1) if $1 \leq p \leq \infty$ and $1 \leq j \leq n$ the operators

$$
\frac{\partial}{\partial x_{j}}(\mathscr{L}-\lambda I)^{-1} \text { and }(\mathscr{L}-\lambda I)^{-1} b \frac{\partial}{\partial x_{j}} \text { are bounded on } L^{p}\left(\mathrm{R}^{n}\right) \text {, }
$$

(2) if $1<p<\infty$ and $1 \leq j, k \leq n$ the operators

$$
\begin{aligned}
& \frac{\partial^{2}}{\partial x_{j} \partial x_{k}}(\mathscr{L}-\lambda I)^{-1}, \frac{\partial}{\partial x_{j}}(\mathscr{L}-\lambda I)^{-1} b \frac{\partial}{\partial x_{k}} \text { and } \\
& (\mathscr{L}-\lambda I)^{-1} b \frac{\partial^{2}}{\partial x_{j} \partial x_{k}} \quad \text { are bounded on } L^{p}\left(\mathrm{R}^{n}\right)
\end{aligned}
$$

with norms depending solely on $\|b\|_{\infty},\left\|\frac{1}{b}\right\|_{\infty}, \mu$ and $\omega$; and also on $p$ in (2).

Proof. Use the expansion in the proof of Proposition 1.1. To obtain (1) observe that for any $1 \leq j \leq n, \frac{\partial}{\partial x_{j}}(-\Delta+\rho I)^{-1}=(-\Delta+\rho I)^{-1} \frac{\partial}{\partial x_{j}}$ is a bounded operator for any $1 \leq p \leq \infty$, since it has a convolution kernel whose $L^{1}\left(\mathrm{R}^{n}\right)$-norm is bounded by $\frac{c}{\sqrt{\rho}}$. For (2) observe that for any $1 \leq j$, 
$k \leq n, \frac{\partial^{2}}{\partial x_{j} \partial x_{k}}(-\Delta+\rho I)^{-1}=(-\Delta+\rho I)^{-1} \frac{\partial^{2}}{\partial x_{j} \partial x_{k}}$ is a bounded operator for any $1<p<\infty$. This follows from the fact that $-\Delta(-\Delta+\rho I)^{-1}=$ $I-\rho(-\Delta+\rho I)^{-1}: L^{p} \rightarrow L^{p}$ for all $1 \leq p \leq \infty$ and that the Riesz transforms are bounded in $L^{p}$ for any $1<p<\infty$ [38, pp. 130-138].

Corollary 1.3. Let $\mu>\omega$. If $p=2$, Proposition 1.2 remains valid for $\lambda \notin S_{\mu+},|\lambda|=1$. The constants depend on $\|b\|_{\infty},\left\|\frac{1}{b}\right\|_{\infty}, \mu$ and $\omega$.

Proof. Let $|\arg \lambda|>\omega,|\lambda|=1$. By the resolvent equation

$$
(\mathscr{L}-\lambda I)^{-1}-(\mathscr{L}+I)^{-1}=(1+\lambda)(\mathscr{L}+I)^{-1}(\mathscr{L}-\lambda I)^{-1},
$$

we have that

$$
\begin{aligned}
(\mathscr{L}-\lambda I)^{-1} & =(\mathscr{L}+I)^{-1}\left\{I+(1+\lambda)(\mathscr{L}-\lambda I)^{-1}\right\} \\
& =\left\{I+(1+\lambda)(\mathscr{L}-\lambda I)^{-1}\right\}(\mathscr{L}+I)^{-1} .
\end{aligned}
$$

Part (1), as well as the boundedness of the first and last operators in (2), now follows from Proposition 1.1 (1) and Proposition 1.2. To handle the middle operator, write

$$
\begin{aligned}
\frac{\partial}{\partial x_{j}}(\mathscr{L}-\lambda I)^{-1} b \frac{\partial}{\partial x_{k}} & =\frac{\partial}{\partial x_{j}}(\mathscr{L}+I)^{-1} b \frac{\partial}{\partial x_{k}} \\
& +(1+\lambda)\left\{\frac{\partial}{\partial x_{j}}(\mathscr{L}+I)^{-1}\right\}\left\{(\mathscr{L}-\lambda I)^{-1} b \frac{\partial}{\partial x_{k}}\right\}
\end{aligned}
$$

and apply what has just been proved, along with Proposition 1.2.

Lemma 1.4. Let $1<p<\infty, \mu>\omega$ and $\lambda \notin S_{\left(\frac{\pi}{2}+\mu\right)+},|\lambda|=1$. Then provided $\frac{1}{q} \geq \frac{1}{p}-\frac{2}{n}$ if $2 p<n$ or $p \leq q<\infty$ if $2 p \geq n$, we have

$(-b \Delta-\lambda I)^{-1}: L^{p} \rightarrow W^{2, p} \hookrightarrow L^{q} \quad$ and $\quad b(-\Delta b-\lambda I)^{-1}: L^{p} \rightarrow W^{2, p} \hookrightarrow L^{q}$ with constants depending on $p, \omega,\|b\|_{\infty}$ and $\left\|\frac{1}{b}\right\|_{\infty}$ only.

Proof. Apply the Sobolev embedding theorem together with Proposition 1.1 (2), Proposition 1.2 (2) and a duality argument.

Proposition 1.5. Let $2 \leq r<\infty$ and $\mu>\omega$. If $N \geq 1+\frac{n}{2}\left(\frac{1}{2}-\frac{1}{r}\right)$ and $|\arg \lambda| \geq \mu,|\lambda|=1$ then,

$$
(-b \Delta-\lambda I)^{-N}: L^{2} \rightarrow W^{2, r} \quad \text { and } \quad b(-\Delta b-\lambda I)^{-N}: L^{2} \rightarrow W^{2, r}
$$

with constants depending on $r, \omega, \mu,\|b\|_{\infty}$ and $\left\|\frac{1}{b}\right\|_{\infty}$.

Proof. Assume first that $\lambda=-1$. Then, given $2 \leq r<\infty$, choose a sequence $2=r_{1}, r_{2}, \ldots, r_{N-1}, r_{N}=r$ such that $\frac{1}{r_{m+1}} \geq \frac{1}{r_{m}}-\frac{2}{n}$ for $m=1,2, \ldots$, 
$N-1$, which is possible because $\frac{1}{2}-\frac{1}{r}=\sum_{m=1}^{N-1}\left(\frac{1}{r_{m}}-\frac{1}{r_{m+1}}\right) \leq \frac{2}{n}(N-1)$. Apply Lemma $1.4(N-1)$ times with $p=r_{m}, \quad q=r_{m+1}$, and $m=1,2, \ldots, N-1$ together with the boundedness of $(-b \Delta+I)^{-1}$ and $b(-\Delta b+I)^{-1}$ from $L^{r}$ into $W^{2, r}$ to reach the desired conclusion.

To extend the result to any $|\arg \lambda| \geq \mu,|\lambda|=1$, use the resolvent equation

$$
(-b \Delta-\lambda I)^{-N}=(-b \Delta+I)^{-N}\left\{I+(1+\lambda)(-b \Delta-\lambda I)^{-1}\right\}^{N} .
$$

Similarly,

$$
b(-\Delta b-\lambda I)^{-N}=b(-\Delta b+I)^{-N}\left\{I+(1+\lambda)(-\Delta b-\lambda I)^{-1}\right\}^{N} .
$$

The result now follows from the case $\lambda=-1$ and Proposition 1.1 (1).

\section{§2. Regularity and kernel estimates for the iterated resolvent}

Let us obtain pointwise bounds and fast decaying estimates on the kernel of the iterated resolvent of $-b \Delta$ times $b$, as well as on its gradient. In the next section we obtain similar estimates for the heat kernel of $-b \Delta$ times $b$ as well as for the Green's function times $b$.

Theorem 2.1. Let $0<\eta<1, M \geq 0$ and $N \geq 1+\frac{n}{4}$. Then the distributional kernel $G_{\lambda}(x, y)$ of $(\mathscr{L}-\lambda I)^{-2 N} b$ belongs to $C^{1, \eta}\left(\mathrm{R}^{n} \times \mathrm{R}^{n}\right)$ for all $\lambda \notin S_{\omega+}$. Moreover, given $\mu>\omega$ there exists $C=C\left(\omega, \mu,\|b\|_{\infty},\left\|\frac{1}{b}\right\|_{\infty}, M, \eta\right)>0$ such that for all $\lambda \notin S_{\mu+}$ and for all $x, y \in \mathbf{R}^{n}$, the following estimates are satisfied,

$$
\begin{aligned}
\left|G_{\lambda}(x, y)\right| & +|\lambda|^{-\frac{1}{2}}\left|\nabla_{x} G_{\lambda}(x, y)\right|+|\lambda|^{-\frac{1}{2}}\left|\nabla_{y} G_{\lambda}(x, y)\right| \\
& \leq C \frac{|\lambda|^{\frac{n}{2}-2 N}}{1+\left(|\lambda|^{\frac{1}{2}}|x-y|\right)^{M}} \\
\mid \nabla G_{\lambda}(x+h, y) & -\nabla G_{\lambda}(x, y)|+| \nabla G_{\lambda}(x, y+h)-\nabla G_{\lambda}(x, y) \mid \\
& \leq C \frac{|\lambda|^{\frac{n+1+\eta}{2}-2 N}|h|^{\eta}}{1+\left(|\lambda|^{\frac{1}{2}}|x-y|\right)^{M}}
\end{aligned}
$$

when $2|h| \leq|x-y|$, where $\nabla=\left(\nabla_{x}, \nabla_{y}\right)$.

Remark. The polynomial decay can be improved to exponential decay by keeping track of the dependence of the constants on $M$. In particular, it is not difficult to check that the constant $C$ in Lemma 2.4 below has the form $c_{1} M !\left(c_{2}\right)^{M}$. This observation is due to Auscher. We shall not pursue this point here, since polynomial decay is sufficient for our purposes of proving bounds on the functional calculus of $-b \Delta$.

Proof $(M=0)$. We initially prove the bounds when $M=0$. 
Assume for the moment that $\lambda \notin S_{\omega+}$ satisfies $|\lambda|=1$. Given $0<\eta<1$, choose $2 \leq r<\infty$ so that $r>\frac{n}{1-\eta}$ and choose $N$ in Proposition 1.5 to be any $N \geq 1+\frac{n}{4}$. Thus by Proposition 1.5, our choice of $r$ and the Sobolev embeddings we have that

$$
(-b \Delta-\lambda I)^{-N}: L^{2}\left(\mathrm{R}^{n}\right) \rightarrow W^{2, r}\left(\mathrm{R}^{n}\right) \hookrightarrow C^{1, \eta}\left(\mathrm{R}^{n}\right) \hookrightarrow L^{\infty}\left(\mathrm{R}^{n}\right)
$$

and

$$
b(-\Delta b-\lambda I)^{-N}: L^{2}\left(\mathrm{R}^{n}\right) \rightarrow W^{2, r}\left(\mathrm{R}^{n}\right) \hookrightarrow C^{1, \eta}\left(\mathrm{R}^{n}\right) \hookrightarrow L^{\infty}\left(\mathrm{R}^{n}\right) .
$$

Furthermore, using the dual pairing $\langle u, v\rangle=\int_{\mathrm{R}^{n}} u(x) v(x) d x$ between $L^{\infty}\left(\mathrm{R}^{n}\right)$ and $L^{1}\left(\mathrm{R}^{n}\right)$ we also get that $(-b \Delta-\lambda I)^{-N} b: L^{1}\left(\mathrm{R}^{n}\right) \rightarrow L^{2}\left(\mathrm{R}^{n}\right)$. Hence,

$$
(-b \Delta-\lambda I)^{-2 N} b: L^{1}\left(\mathrm{R}^{n}\right) \rightarrow W^{2, r} \hookrightarrow C^{1, \eta} \hookrightarrow L^{\infty}\left(\mathrm{R}^{n}\right),
$$

from which we conclude that $G_{\lambda} \in L^{\infty}\left(\mathrm{R}^{n} \times \mathrm{R}^{n}\right)$. Moreover, by the embeddings above we also have that $G_{\lambda}(\cdot, y) \in C^{1, \eta}\left(\mathrm{R}^{n}\right)$ for a.a. $y \in \mathrm{R}^{n}$ and since by a duality argument $b(-\Delta b-\lambda I)^{-2 N}: L^{1}\left(\mathbf{R}^{n}\right) \rightarrow C^{1, \eta}$, we also have that $G_{\lambda}(x, \cdot) \in C^{1, \eta}\left(\mathrm{R}^{n}\right)$ for a.a. $x \in \mathrm{R}^{n}$. Together, these imply that $G_{\lambda} \in C^{1, \eta}\left(\mathrm{R}^{n} \times \mathrm{R}^{n}\right)$.

The bounds on $G_{\lambda}$ and its derivatives depend on $\omega, \mu,\|b\|_{\infty}$ and $\left\|\frac{1}{b}\right\|_{\infty}$ but are independent of $\arg \lambda$.

To remove the assumption $|\lambda|=1$ do a rescaling argument. Given any $\lambda$ with $|\arg \lambda| \geq \mu$, write $\lambda=r \zeta$ where $|\arg \zeta| \geq \mu$ and $|\zeta|=1$. Let $X=\sqrt{r} x$ and $Y=\sqrt{r} y$ and write $f_{r}(X)=f\left(\frac{X}{\sqrt{r}}\right)$. Then

$$
\begin{aligned}
\int G_{\lambda}(x, y) u(y) d y & =\left(b(-\Delta)_{x}-\lambda I\right)^{-2 N} b(x) u(x) \\
& =r^{-2 N}\left(b_{r}(X)(-\Delta)_{X}-\zeta I\right)^{-2 N} b_{r}(X) u_{r}(X) \\
& =r^{-2 N} \int G_{\zeta}^{(r)}(X, Y) u_{r}(Y) d Y \\
& =\int r^{\frac{n}{2}-2 N} G_{\zeta}^{(r)}(\sqrt{r} x, \sqrt{r} y) u(y) d y
\end{aligned}
$$

where $G_{\zeta}^{(r)}$ is the kernel of $\left(-b_{r} \Delta-\zeta I\right)^{-2 N} b_{r}$. Therefore $G_{\lambda}(x, y)=$ $r^{\frac{n}{2}-2 N} G_{\zeta}^{(r)}(x, y)$. Observe that $b$ and $\frac{1}{b}$ are bounded $\omega$-accretive multiplication operators which satisfy scale invariant conditions, so $G_{\zeta}^{(r)}$ satisfies the same estimates as $G_{\zeta}$. Hence $\left|G_{\lambda}(x, y)\right| \leq c|\lambda|^{\frac{n}{2}-2 N}$, and the other required bounds hold too.

The remainder of this section will be spent in proving that the kernels and their derivatives decay as $|x-y| \rightarrow \infty$ in order to complete the proof of 
Theorem 2.1 for $M>0$. To that effect we derive operator bounds on the commutators $\left[g,(\mathscr{L}-\lambda I)^{-N}\right]$ defined by

$$
\left[g,(\mathscr{L}-\lambda I)^{-N}\right] u=g(\mathscr{L}-\lambda I)^{-N} u-(\mathscr{L}-\lambda I)^{-N}(g u)
$$

in terms of $\|\nabla g\|_{\infty}$ when $g \in C_{0}^{2}\left(\mathrm{R}^{n}\right)$. No assumptions are ever made on $\|g\|_{\infty}$ or on the size of its support.

In what follows the summation convention is used.

Lemma 2.2. Let $g \in C_{0}^{2}\left(\mathrm{R}^{n}\right)$. For any $\mu>\omega$ there exists a constant $c=c(\mu)>0$ such that for any $1 \leq p \leq \infty$ and any $\lambda \notin S_{(\pi / 2+\mu)+}, \quad|\lambda|=1$,

$$
\left\|\left[g,(\mathscr{L}-\lambda I)^{-1}\right]\right\|_{p, p}+\left\|\left[g, b(-\Delta b-\lambda I)^{-1}\right]\right\|_{p, p} \leq c\|\nabla g\|_{\infty} .
$$

Proof. Observe that, formally,

$$
\begin{aligned}
{\left[g,(\mathscr{L}-\lambda I)^{-1}\right] } & =-(\mathscr{L}-\lambda I)^{-1}[g, \mathscr{L}](\mathscr{L}-\lambda I)^{-1} \\
& =(\mathscr{L}-\lambda I)^{-1} b \frac{\partial}{\partial x_{j}} \frac{\partial g}{\partial x_{j}}(\mathscr{L}-\lambda I)^{-1} \\
& +(\mathscr{L}-\lambda I)^{-1} b \frac{\partial g}{\partial x_{j}} \frac{\partial}{\partial x_{j}}(\mathscr{L}-\lambda I)^{-1} .
\end{aligned}
$$

Hence Proposition 1.1 and 1.2 yield the desired results.

To justify the formulae in the above expression, note that if $v \in L^{p}\left(\mathrm{R}^{n}\right)$, then $u=(\mathscr{L}-\lambda I)^{-1} v \in W^{2, p}\left(\mathrm{R}^{n}\right)$ and

$$
\begin{aligned}
{[g, \mathscr{L}] u=[g,(\mathscr{L}-\lambda I)] u } & =(g(\mathscr{L}-\lambda I)-(\mathscr{L}-\lambda I) g) u \\
& =-g b \Delta u+b \Delta(g u)=b \frac{\partial g}{\partial x_{j}} \frac{\partial u}{\partial x_{j}}+b \frac{\partial}{\partial x_{j}}\left(\frac{\partial g}{\partial x_{j}} u\right) .
\end{aligned}
$$

Similar reasoning yields the estimate for $\left[g, b(-\Delta b-\lambda I)^{-1}\right]$.

Let us comment further that, if $g \in C_{0}^{2}\left(\mathrm{R}^{n}\right)$ and $u \in W^{2, p}\left(\mathrm{R}^{n}\right)$, then

$$
[g[g, \mathscr{L}]] u=-2 b\left(\frac{\partial g}{\partial x_{j}}\right)^{2} u \text { and }[g[g[g, \mathscr{L}]]] u=0 .
$$

Let us denote by $\delta$ the derivation, $\delta(A)=[g, A]$ mapping the space of bounded linear operators on $L^{p}\left(\mathrm{R}^{n}\right)$ to itself. Observe that $\delta(A B)=A \delta(B)+$ $B \delta(A)$ and that $\delta\left(A^{-1}\right)=-A^{-1} \delta(A) A^{-1}$. We also define 


$$
\delta^{k}(\mathscr{L})= \begin{cases}b\left(\frac{\partial}{\partial x_{j}}\left(\frac{\partial g}{\partial x_{j}}\right)+\frac{\partial g}{\partial x_{j}} \frac{\partial}{\partial x_{j}}\right) & \text { if } k=1 \\ -2 b\left(\frac{\partial g}{\partial x_{j}}\right)^{2} & \text { if } k=2 \\ 0 & \text { if } k \geq 3 .\end{cases}
$$

The derivation formula for $\delta(A B)$ continues to hold when $\mathscr{L}$ replaces $A$ or $B$, provided it makes sense. In particular the following statements hold.

Lemma 2.3. For $\delta$ defined as above,

$$
\begin{aligned}
\delta\left((\mathscr{L}-\lambda I)^{-1}\right) & =-(\mathscr{L}-\lambda I)^{-1} \delta(\mathscr{L})(\mathscr{L}-\lambda I)^{-1} \\
\delta\left(\delta(\mathscr{L})(\mathscr{L}-\lambda I)^{-1}\right) & =\delta^{2}(\mathscr{L})(\mathscr{L}-\lambda I)^{-1}+\delta(\mathscr{L}) \delta\left((\mathscr{L}-\lambda I)^{-1}\right) .
\end{aligned}
$$

Lemma 2.4. For any $\mu>\omega, M \geq 1$ and $1<p<\infty$ there exists a constant $C=C\left(p, \mu,\|b\|_{\infty},\left\|\frac{1}{b}\right\|_{\infty}, M\right)>0$ such that for any $\lambda \notin S_{(\pi / 2+\mu)+},|\lambda|=1$,

$$
\left\|\delta^{M}\left((\mathscr{L}-\lambda I)^{-1}\right)\right\|_{p, p}+\left\|\delta^{M}\left(b(-\Delta b-\lambda I)^{-1}\right)\right\|_{p, p} \leq C\|\nabla g\|_{\infty}^{M} .
$$

Proof. Lemma 2.2 gives the case $M=1$. For $M=2$ apply Lemma 2.3 to obtain

$$
\begin{aligned}
\delta^{2}\left((\mathscr{L}-\lambda I)^{-1}\right)= & 2(\mathscr{L}-\lambda I)^{-1} \delta(\mathscr{L})(\mathscr{L}-\lambda I)^{-1} \delta(\mathscr{L})(\mathscr{L}-\lambda I)^{-1} \\
& -(\mathscr{L}-\lambda I)^{-1} \delta^{2}(\mathscr{L})(\mathscr{L}-\lambda I)^{-1} \\
= & 2(\mathscr{L}-\lambda I)^{-1} b \frac{\partial}{\partial x_{j}} \frac{\partial g}{\partial x_{j}}(\mathscr{L}-\lambda I)^{-1} b \frac{\partial}{\partial x_{j}} \frac{\partial g}{\partial x_{j}}(\mathscr{L}-\lambda I)^{-1} \\
& +2(\mathscr{L}-\lambda I)^{-1} b \frac{\partial}{\partial x_{j}} \frac{\partial g}{\partial x_{j}}(\mathscr{L}-\lambda I)^{-1} b \frac{\partial g}{\partial x_{j}} \frac{\partial}{\partial x_{j}}(\mathscr{L}-\lambda I)^{-1} \\
& +2(\mathscr{L}-\lambda I)^{-1} b \frac{\partial g}{\partial x_{j}} \frac{\partial}{\partial x_{j}}(\mathscr{L}-\lambda I)^{-1} b \frac{\partial}{\partial x_{j}} \frac{\partial g}{\partial x_{j}}(\mathscr{L}-\lambda I)^{-1} \\
& +2(\mathscr{L}-\lambda I)^{-1} b \frac{\partial g}{\partial x_{j}} \frac{\partial}{\partial x_{j}}(\mathscr{L}-\lambda I)^{-1} b \frac{\partial g}{\partial x_{j}} \frac{\partial}{\partial x_{j}}(\mathscr{L}-\lambda I)^{-1} \\
& +2(\mathscr{L}-\lambda I)^{-1} b\left(\frac{\partial g}{\partial x_{j}}\right)^{2}(\mathscr{L}-\lambda I)^{-1}
\end{aligned}
$$

which is bounded on $L^{p}\left(\mathrm{R}^{n}\right)$ for $1<p<\infty$ by Proposition 1.2.

Finally, if $M \geq 3, \delta^{M}\left((\mathscr{L}-\lambda I)^{-1}\right)$ is a linear combination of terms of the form $(\mathscr{L}-\lambda I)^{-1} \delta^{k_{1}}(\mathscr{L})(\mathscr{L}-\lambda I)^{-1} \delta^{k_{2}}(\mathscr{L})(\mathscr{L}-\lambda I)^{-1} \ldots(\mathscr{L}-\lambda I)^{-1} \delta^{k_{m}}(\mathscr{L})$. $(\mathscr{L}-\lambda I)^{-1}$ where $k_{1}+k_{2}+\cdots+k_{m}=M, 1 \leq k_{j} \leq 2$ for all $j=1, \ldots, m$ and $\frac{M}{2} \leq m \leq M$, since $\delta^{k}(\mathscr{L})=0$ for $k \geq 3$. Hence Lemma 2.3 and Propo- 
sition 1.2 imply once again the desired result since multiplication by $b$ and $\frac{\partial g}{\partial x_{j}}$ define bounded operators on $L^{p}\left(\mathrm{R}^{n}\right)$.

A similar reasoning yields the estimate for $\delta^{M}\left(b(-\Delta b-\lambda I)^{-1}\right)$.

Remark. If in Lemma 2.4 we allowed the estimates to depend on $\|\Delta g\|_{\infty}$ as well as on $\|\nabla g\|_{\infty}$, then the constant $C$ would not depend on $p$ and the results would be also valid for $p=1$ and $p=\infty$.

Corollary 2.5. If $p=2$, Lemma 2.2 and 2.4 are also valid for $\lambda \notin S_{\omega+}$, $|\lambda|=1$.

Proof. By Proposition 1.1 (1) and Corollary 1.3 the same proofs as those of Lemma 2.2 and 2.4, respectively, remain valid in $L^{2}\left(\mathrm{R}^{n}\right)$ for any $|\arg \lambda|>\omega,|\lambda|=1$.

Lemma 2.6. Given $\mu>\omega, M \geq 1$ and $1<p \leq q$ where $\frac{1}{q} \geq \frac{1}{p}-\frac{2}{n}$ if $q<\infty$ and $p>\frac{n}{2}$ if $q=\infty$. Then, there exists a constant $C=C\left(p, \mu,\|b\|_{\infty}\right.$, $\left.\left\|\frac{1}{b}\right\|_{\infty}, M\right)>0$ such that for any $\lambda \notin S_{(\pi / 2+\mu)+},|\lambda|=1$,

$$
\left\|\delta^{M}\left((-b \Delta-\lambda I)^{-1}\right)\right\|_{p, q}+\left\|\delta^{M}\left(b(-\Delta b-\lambda I)^{-1}\right)\right\|_{p, q} \leq C\|\nabla g\|_{\infty}^{M} .
$$

Proof. Write $\delta^{M}\left((-b \Delta-\lambda I)^{-1}\right)$ as in the proof of Lemma 2.4. The desired estimate now follows from Lemma 1.4 and Proposition 1.2 which give

$$
\begin{aligned}
(-b \Delta-\lambda I)^{-1}: L^{p} & \rightarrow L^{q} \\
\frac{\partial}{\partial x_{j}}(-b \Delta-\lambda I)^{-1} \text { and }(-b \Delta-\lambda I)^{-1} b \frac{\partial}{\partial x_{j}}: L^{r} & \rightarrow L^{r} \text { for } 1 \leq r \leq \infty \\
\frac{\partial}{\partial x_{j}}(-b \Delta-\lambda I)^{-1} b \frac{\partial}{\partial x_{k}}: L^{r} & \rightarrow L^{r} \text { for } 1<r<\infty .
\end{aligned}
$$

The estimate for $\delta^{M}\left(b(-\Delta b-\lambda I)^{-1}\right)$ is obtained in a similar way.

Lemma 2.7. For any $\mu>\omega, M \geq 1$ and $1<p<\infty$ there exists a positive constant $C\left(p, \mu,\|b\|_{\infty},\left\|\frac{1}{b}\right\|_{\infty}, M\right)$ such that for any $1 \leq k \leq n$ and $\lambda \notin S_{(\pi / 2+\mu)+},|\lambda|=1$,

$$
\left\|\frac{\partial}{\partial x_{k}} \delta^{M}\left((\mathscr{L}-\lambda I)^{-1}\right)\right\|_{p, p}+\left\|\delta^{M}\left((\mathscr{L}-\lambda I)^{-1} b\right) \frac{\partial}{\partial x_{k}}\right\|_{p, p} \leq C\left(\|\nabla g\|_{\infty}\right)^{M} .
$$

Proof. Write $\delta^{M}\left((\mathscr{L}-\lambda I)^{-1}\right)$ as in the proof of Lemma 2.4. Observe that we always have $(\mathscr{L}-\lambda I)^{-1}$ or $(\mathscr{L}-\lambda I)^{-1} b \frac{\partial}{\partial x_{j}}$ at the far left and $(\mathscr{L}-\lambda I)^{-1}$ or $\frac{\partial}{\partial x_{j}}(\mathscr{L}-\lambda I)^{-1}$ at the far right of each term in the linear combination. Conclude by applying Proposition 1.2. 
Lemma 2.8. Let $2 \leq r<\infty, \mu>\omega$ and $N \geq 1+\frac{n}{4}$. For any $M \geq 1$ and any $|\arg \lambda| \geq \mu,|\lambda|=1$,

$\delta^{M}\left((-b \Delta-\lambda I)^{-N}\right): L^{2} \rightarrow W^{1, r} \quad$ and $\quad \delta^{M}\left(b(-\Delta b-\lambda I)^{-N}\right): L^{2} \rightarrow W^{1, r}$ with norms bounded by a positive constant $C\left(\omega, \mu,\|b\|_{\infty},\left\|\frac{1}{b}\right\|_{\infty}, M\right)$ times $\|\nabla g\|_{\infty}^{M}$

Proof. Let us first assume that $\lambda=-1$ and recall that $\mathscr{L}=-b \Delta$. For convenience let us define $\delta^{0}\left((\mathscr{L}+I)^{-1}\right)=(\mathscr{L}+I)^{-1}$. By the properties of $\delta$, we can write

$$
\begin{gathered}
\delta^{M}\left((\mathscr{L}+I)^{-N}\right)=\sum C_{M_{1} M_{2} \ldots M_{N}} \delta^{M_{1}}\left((\mathscr{L}+I)^{-1}\right) \delta^{M_{2}}\left((\mathscr{L}+I)^{-1}\right) \ldots \\
\ldots \delta^{M_{N}}\left((\mathscr{L}+I)^{-1}\right)
\end{gathered}
$$

where the sum is over all $N$-tuples, $\left(M_{1}, M_{2}, \ldots, M_{N}\right)$ such that $0 \leq M_{j} \leq M$ and $M_{1}+M_{2}+\ldots M_{N}=M$. The constant $C_{M_{1} M_{2} \ldots M_{N}}=\frac{M !}{M_{1} ! M_{2} ! \ldots M_{N} !}$ is computed in a similar way as in the multinomial theorem.

Given $2 \leq r<\infty$ and $N \geq 1+\frac{n}{4}$ proceed as in Proposition 1.5. Choose a sequence $2=r_{1} \leq r_{2} \leq \ldots, r_{N}=r$ such that by applying Lemma 1.4 and Lemma $2.6(N-1)$ times with $p=r_{m}$ and $q=r_{m+1}$ we have

$$
\delta^{M}\left((\mathscr{L}+I)^{-N}\right): L^{2}\left(\mathrm{R}^{n}\right) \rightarrow W^{1, r}\left(\mathrm{R}^{n}\right)
$$

with norm bounded by a positive constant $C\left(\omega, \mu,\|b\|_{\infty},\left\|\frac{1}{b}\right\|_{\infty}, M\right)$ times $\|\nabla g\|_{\infty}^{M}$. We have used the fact that by Lemma 2.4 and Lemma 2.7 $\delta^{M_{1}}\left((\mathscr{L}+I)^{-1}\right): L^{r}\left(\mathrm{R}^{n}\right) \rightarrow W^{1, r}\left(\mathrm{R}^{n}\right)$ with norm bounded by a constant times $\|\nabla g\|_{\infty}^{M_{1}}$.

For a general $\lambda \notin S_{\omega+},|\lambda|=1$ recall that by the resolvent equation,

$$
(\mathscr{L}-\lambda I)^{-N}=(\mathscr{L}+I)^{-N}\left\{I+(1+\lambda)(\mathscr{L}-\lambda I)^{-1}\right\}^{N} .
$$

Therefore,

$\delta^{M}\left((\mathscr{L}-\lambda I)^{-N}\right)=\sum_{m=0}^{M}\left(\begin{array}{c}M \\ m\end{array}\right) \delta^{m}\left((\mathscr{L}+I)^{-N}\right) \delta^{M-m}\left(\left\{I+(1+\lambda)(\mathscr{L}-\lambda I)^{-1}\right\}^{N}\right)$.

By Corollary 2.5 and the properties of $\delta$,

$$
\delta^{M-m}\left(\left\{I+(1+\lambda)(\mathscr{L}-\lambda I)^{-1}\right\}^{N}\right): L^{2}\left(\mathrm{R}^{n}\right) \rightarrow L^{2}\left(\mathrm{R}^{n}\right) .
$$

The desired result then follows from the previous case where $\lambda=-1$.

Analogous reasoning yields $\delta^{M}\left(b(-\Delta b-\lambda I)^{-N}\right): L^{2}\left(\mathrm{R}^{n}\right) \rightarrow W^{1, r}\left(\mathrm{R}^{n}\right)$.

Corollary 2.9. Let $\mu>\omega$ and $N \geq 1+\frac{n}{4}$. For any $M \geq 1$ and any $|\arg \lambda| \geq \mu,|\lambda|=1$, 


$$
\delta^{M}\left((-b \Delta-\lambda I)^{-2 N} b\right): L^{1}\left(\mathrm{R}^{n}\right) \rightarrow L^{\infty}\left(\mathrm{R}^{n}\right)
$$

with norm bounded by $\|\nabla g\|_{\infty}^{M}$ times a constant depending on $\omega, \mu,\|b\|_{\infty}$, $\left\|\frac{1}{b}\right\|_{\infty}$ and $M$.

Proof. By Lemma 2.8 applied with $r>n$ and the Sobolev embeddings,

$$
\delta^{M}\left((-b \Delta-\lambda I)^{-N}\right): L^{2}\left(\mathrm{R}^{n}\right) \rightarrow W^{1, r}\left(\mathrm{R}^{n}\right) \hookrightarrow L^{\infty}\left(\mathrm{R}^{n}\right)
$$

and

$$
\delta^{M}\left(b(-\Delta b-\lambda I)^{-N}\right): L^{2}\left(\mathrm{R}^{n}\right) \rightarrow W^{1, r}\left(\mathrm{R}^{n}\right) \hookrightarrow L^{\infty}\left(\mathrm{R}^{n}\right) .
$$

Moreover, by duality, Lemma 2.8 yields $\delta^{M}\left((-b \Delta-\lambda I)^{-N} b\right): L^{1}\left(\mathrm{R}^{n}\right) \rightarrow$ $L^{2}\left(\mathrm{R}^{n}\right)$. Hence,

$$
\delta^{M}\left((-b \Delta-\lambda I)^{-2 N} b\right): L^{1}\left(\mathrm{R}^{n}\right) \rightarrow L^{\infty}\left(\mathrm{R}^{n}\right)
$$

since

$$
\delta^{M}\left((-b \Delta-\lambda I)^{-2 N} b\right)=\sum_{m=0}^{M}\left(\begin{array}{c}
M \\
m
\end{array}\right) \delta^{m}\left((-b \Delta-\lambda I)^{-N}\right) \delta^{M-m}\left((-b \Delta-\lambda I)^{-N} b\right) .
$$

We are now in a position to prove the decay bound on $G_{\lambda}$.

Proposition 2.10. Let $\mu>\omega$ and $N \geq 1+\frac{n}{4}$. Then for any $M \geq 1$ there exists a constant $C=C\left(\omega, \mu,\|b\|_{\infty},\left\|\frac{1}{b}\right\|_{\infty}, M\right)>0$ such that the distribution kernel $G_{\lambda}(x, y)$ of $(\mathscr{L}-\lambda I)^{-2 N} b$ satisfies

$$
\left|G_{\lambda}(x, y)\right| \leq C \frac{|\lambda|^{\frac{n}{2}-2 N}}{1+\left(|\lambda|^{\frac{1}{2}}|x-y|\right)^{M}}, \quad \lambda \notin S_{\mu+} .
$$

Proof. By the proof of Theorem 2.1 we have that for any $\lambda \notin S_{\mu+}$,

$$
\left|G_{\lambda}(x, y)\right| \leq C|\lambda|^{\frac{n}{2}-2 N} .
$$

Assume $|\lambda|=1$. By Corollary 2.9 there exists another positive constant $C$ such that

$$
|g(x)-g(y)|^{M}\left|G_{\lambda}(x, y)\right| \leq C\|\nabla g\|_{\infty}^{M},
$$

for any $g \in C_{0}^{2}\left(\mathrm{R}^{n}\right)$. Thus, given $x, y$ choose $g \in C_{0}^{2}\left(\mathrm{R}^{n}\right)$ such that $|g(x)-g(y)|=|x-y|$ and $\|\nabla g\|_{\infty} \leq 2$. It follows that

$$
\left|G_{\lambda}(x, y)\right| \leq \frac{C}{|x-y|^{M}} .
$$

By a rescaling argument, we can remove the condition $|\lambda|=1$ to obtain 


$$
\left|G_{\lambda}(x, y)\right| \leq \frac{C|\lambda|^{\frac{n}{2}-2 N}}{\left(|\lambda|^{\frac{1}{2}}|x-y|\right)^{M}} .
$$

REMARK. We got the idea of using commutators to prove the decay of the kernels from Auscher. It is closely related to the method developed by Davies to derive pointwise bounds with exponential decay on heat kernels [18] by letting $g$ depend on $x$ and $y$.

To complete the proof of Theorem 2.1 it remains to prove decay of the gradient of $G_{\lambda}(x, y)$ with respect to both $x$ and $y$. From these estimates, decay for the $C^{1, \eta}$ bounds of $G_{\lambda}(x, y)$ will easily follows as indicated in Corollary 2.12 below.

Proposition 2.11. Let $\mu>\omega$ and $N \geq 1+\frac{n}{4}$. Then for any $M \geq 1$ there exists a constant $C=C\left(\omega, \mu,\|b\|_{\infty},\left\|\frac{1}{b}\right\|_{\infty}, M\right)>0$ such that

$$
\left|\nabla_{x} G_{\lambda}(x, y)\right|+\left|\nabla_{y} G_{\lambda}(x, y)\right| \leq C \frac{|\lambda|^{\frac{n+1}{2}-2 N}}{1+\left(|\lambda|^{\frac{1}{2}}|x-y|\right)^{M}}, \quad \lambda \notin S_{\mu+} .
$$

Proof. Assume that $\lambda \notin S_{\mu+}$ and $|\lambda|=1$. It then suffices to consider $x$ and $y$ satisfying $|x-y| \geq 2$. For $1 \leq j \leq n$, let $e_{j}$ denote the unit vector in the $j^{\prime}$ th direction. By the case $M=0$ of Theorem 2.1, we have

$$
\left|\frac{\partial G_{\lambda}}{\partial x_{j}}\left(x+\tau e_{j}, y\right)-\frac{\partial G_{\lambda}}{\partial x_{j}}(x, y)\right| \leq C|\tau|^{\frac{1}{2}}
$$

when $|\tau| \leq 1$. On writing $\frac{\partial G_{\lambda}}{\partial x_{j}}(x, y)=Y e^{i \theta}$ with $Y=\left|\frac{\partial G_{\lambda}}{\partial x_{j}}(x, y)\right| \geq 0$, it follows that

$$
\operatorname{Re}\left\{e^{-i \theta} \frac{\partial G_{\lambda}}{\partial x_{j}}\left(x+\tau e_{j}, y\right)\right\} \geq Y-C|\tau|^{\frac{1}{2}} \geq \frac{1}{2} Y
$$

provided $|\tau| \leq \tau_{0}=\min \left\{\frac{Y^{2}}{4 C^{2}}, 1\right\}$. Therefore

$$
\left|\int_{0}^{ \pm \tau_{0}} \frac{d G_{\lambda}}{d \tau}\left(x+\tau e_{j}, y\right) d \tau\right| \geq \operatorname{Re}\left\{e^{-i \theta} \int_{0}^{ \pm \tau_{0}} \frac{\partial G_{\lambda}}{\partial x_{j}}\left(x+\tau e_{j}, y\right) d \tau\right\} \geq \frac{1}{2} Y \tau_{0}
$$

so that

$$
\begin{aligned}
\frac{1}{2} Y \tau_{0} & \leq\left|G_{\lambda}\left(x \pm \tau_{0} e_{j}, y\right)-G_{\lambda}(x, y)\right| \\
& \leq C \frac{1}{1+\left|x \pm \tau_{0} e_{j}-y\right|^{3 M}}+C \frac{1}{1+|x-y|^{3 M}}
\end{aligned}
$$

by Proposition 2.10. Now, for one choice of sign, $\left|x \pm \tau_{0} e_{j}-y\right| \geq|x-y|$, so we get 


$$
\min \left\{\frac{Y^{3}}{4 C^{2}}, Y\right\}=Y \tau_{0} \leq 4 C \frac{1}{1+|x-y|^{3 M}} .
$$

Hence

$$
\left|\frac{\partial G_{\lambda}}{\partial x_{j}}(x, y)\right|=Y \leq C \frac{1}{1+|x-y|^{M}}
$$

(for a new constant $C$ ) as required. A rescaling argument gives the result for all $\lambda \notin S_{\mu+}$. A similar argument gives the estimate for $\nabla_{y} G_{\lambda}(x, y)$.

REMark. Proposition 2.11 can also be proved by first deriving the following commutator bound. Let $\mu>\omega$ and $N \geq 1+\frac{n}{4}$. For any $M \geq 1$ and $|\arg \lambda| \geq \mu,|\lambda|=1$,

$$
\delta^{M}\left(\frac{\partial}{\partial x_{j}}(-b \Delta-\lambda I)^{-2 N} b\right): L^{1}\left(\mathrm{R}^{n}\right) \rightarrow L^{\infty}\left(\mathrm{R}^{n}\right)
$$

with norm bounded by $\|\nabla g\|_{\infty}^{M-1}\left(\|\nabla g\|_{\infty}+\|\Delta g\|_{\infty}\right)$ times a constant depending on $\omega, \mu,\|b\|_{\infty},\left\|\frac{1}{b}\right\|_{\infty}$ and $M$. The same is true for $\delta^{M}\left(\frac{\partial}{\partial x_{j}} b(-\Delta b-\lambda I)^{-2 N}\right)$.

Corollary 2.12. Let $\mu>\omega$ and $N \geq 1+\frac{n}{4}$. Then for any $M \geq 1$ and any $0<\eta<1$ there exists a constant $C=C\left(\omega, \mu,\|b\|_{\infty},\left\|\frac{1}{b}\right\|_{\infty}, M, \eta\right)>0$ such that,

$$
\begin{aligned}
& \left|\nabla_{x} G_{\lambda}(x+h, y)-\nabla_{x} G_{\lambda}(x, y)\right|+\left|\nabla_{x} G_{\lambda}(x, y+h)-\nabla_{x} G_{\lambda}(x, y)\right| \\
& \leq C \frac{|\lambda|^{\frac{n+1+\eta}{2}-2 N}|h|^{\eta}}{1+\left(|\lambda|^{\frac{1}{2}}|x-y|\right)^{M}}
\end{aligned}
$$

when $2|h| \leq|x-y|$ and for all $\lambda \notin S_{\mu+}$. The same bounds also hold for $\nabla_{y} G_{\lambda}$.

Proof. Given $0<\eta<1$, let $\eta<\alpha<1$ and let $\beta=\frac{\eta}{\alpha}<1$. By Proposition 2.11 applied with $M$ replaced by $M^{\prime}=\frac{M}{1-\beta}$ and by the case $M=0$ of Theorem 2.1, applied with $\alpha$ in place of $\eta$, we have

$$
\begin{aligned}
\left|\nabla_{x} G_{\lambda}(x+h, y)-\nabla_{x} G_{\lambda}(x, y)\right| \leq & \left(\left|\nabla_{x} G_{\lambda}(x+h, y)\right|+\left|\nabla_{x} G_{\lambda}(x, y)\right|\right)^{1-\beta} \\
& \times\left|\nabla_{x} G_{\lambda}(x+h, y)-\nabla_{x} G_{\lambda}(x, y)\right|^{\beta} \\
\leq & \frac{C\left(|\lambda|^{\frac{n+1}{2}-2 N}\right)^{1-\beta}}{1+\left(|\lambda|^{\frac{1}{2}}|x-y|\right)^{M}}\left(|\lambda|^{\frac{n+1+\alpha}{2}-2 N}|h|^{\alpha}\right)^{\beta} \\
\leq & \frac{C|\lambda|^{\frac{n+1+\eta}{2}-2 N}|h|^{\eta}}{1+\left(|\lambda|^{\frac{1}{2}}|x-y|\right)^{M}}
\end{aligned}
$$

for all $x, y$ and $|h| \leq \frac{|x-y|}{2}$. The estimate for variations in $y$ is analogous. 
The proof of Theorem 2.1 is now complete.

\section{§3. Heat kernel estimates and regularity}

The operator $\mathscr{L}$ is of type $\omega$, and thus $e^{-z \mathscr{L}}$ defines a bounded operator on $L^{2}\left(\mathbf{R}^{n}\right)$ if $|\arg z|<\frac{\pi}{2}-\omega$. It is well known that $\left\{e^{-z \mathscr{L}}:|\arg z|<\frac{\pi}{2}-\omega\right\}$ is a holomorphic semigroup acting on $L^{2}\left(\mathrm{R}^{n}\right)$. See, for example, [28, pp. 489493].

Let us prove kernel bounds, together with decay and regularity, for the kernel of $e^{-z \mathscr{L}} b$, namely $K_{z}(x, y)=k_{z}(x, y) b(y)$, where $k_{z}(x, y)$ is the heat kernel of $\mathscr{L}$.

Theorem 3.1. Let $0<\eta<1$. Then, for all $z \in S_{\frac{\pi}{2}-\omega}^{0}$ the distributional kernel of $e^{-z \mathscr{L}}$ b, which we denote by $K_{z}(x, y)$, belongs to $L^{\infty}\left(\mathrm{R}^{n} \times \mathrm{R}^{n}\right)$. Furthermore, $K_{z} \in C^{1, \eta}\left(\mathbf{R}^{n} \times \mathrm{R}^{n}\right)$ and given $\mu>\omega$ and $M \geq 1$, the following estimates are satisfied for all $z \in S_{\frac{\pi}{2}-\mu}^{0}$

$$
\begin{aligned}
\left|K_{z}(x, y)\right| & +|z|^{\frac{1}{2}}\left|\nabla_{x} K_{z}(x, y)\right|+|z|^{\frac{1}{2}}\left|\nabla_{y} K_{z}(x, y)\right| \\
& \leq \frac{C}{|z|^{\frac{n}{2}}} \frac{1}{\left(1+\frac{|x-y|}{|z|^{\frac{1}{2}}}\right)^{M}} \\
\mid \nabla_{x} K_{z}(x+h, y) & -\nabla_{x} K_{z}(x, y)|+| \nabla_{x} K_{z}(x, y+h)-\nabla_{x} K_{z}(x, y) \mid \\
& \leq \frac{C}{|z|^{\frac{n+1+\eta}{2}}} \frac{|h|^{\eta}}{\left(1+\frac{|x-y|}{|z|^{\frac{1}{2}}}\right)^{M}}
\end{aligned}
$$

when $2|h| \leq|x-y|$ with $C=C\left(\omega, \mu,\|b\|_{\infty},\left\|\frac{1}{b}\right\|_{\infty}, M\right)>0$. The same Hölder estimates also hold for $\nabla_{y} K_{z}(x, y)$.

Proof. The argument follows one in [7]. Let $N \geq 1+\frac{n}{4}$ as in Theorem 2.1. Then for $z \in S_{\left(\frac{\pi}{2}-\mu\right)+}^{0}$,

$$
e^{-z \mathscr{L}} b=\frac{(2 N-1) !}{2 \pi i z^{2 N-1}} \int_{\gamma} e^{-z \lambda}(\mathscr{L}-\lambda I)^{-2 N} b d \lambda
$$

where we choose $\gamma$ to be the path of integration given by $\gamma=\gamma_{1} \cup \gamma_{0} \cup \gamma_{-1}$ where $\gamma_{ \pm 1}=\left\{\lambda=r e^{ \pm i \nu}, r \geq R\right\} \quad$ and $\quad \gamma_{0}=\left\{\lambda=R e^{i \theta}, \nu \leq \theta \leq 2 \pi-\nu\right\}$ with $\nu \in(\omega, \mu)$ and $R>0$ to be determined later. Then we have

$$
\left|K_{z}(x, y)\right|=\left|\frac{(2 N-1) !}{2 \pi i z^{2 N-1}} \int_{\gamma} e^{-z \lambda} G_{\lambda}(x, y) d \lambda\right| \leq \frac{(2 N-1) !}{2 \pi|z|^{2 N-1}}\left(I_{1}+I_{0}+I_{-1}\right)
$$

where for $j=1,0,-1$ we have denoted by $I_{j}=\int_{\gamma_{j}}\left|e^{-z \lambda}\right|\left|G_{\lambda}(x, y)\right||d \lambda|$.

By Theorem 2.1 we estimate each term as follows, 


$$
\begin{aligned}
\left|I_{ \pm 1}\right| & \leq \frac{c}{\left(1+R^{\frac{1}{2}}|x-y|\right)^{M}}\left(\int_{R}^{\infty} e^{-c_{\omega, \mu} r|z|} r^{\frac{n}{2}-2 N} d r\right) \\
& \leq \frac{c^{\prime} R^{\frac{n}{2}-2 N}}{|z|\left(1+R^{\frac{1}{2}}|x-y|\right)^{M}} e^{-c_{\omega, \mu} R|z|},
\end{aligned}
$$

since $\operatorname{Re}(z \lambda) \geq c_{\mu, \omega}|z||\lambda|$ for $\lambda \in \gamma_{ \pm 1}$. Also,

$$
\left|I_{0}\right| \leq c \frac{R^{\frac{n}{2}-2 N+1}}{\left(1+R^{\frac{1}{2}}|x-y|\right)^{M}} e^{|z| R} .
$$

Let $R=\frac{1}{|z|}$ to obtain the desired estimates for $K_{z}(x, y)$.

A similar proof yields the bounds on $\nabla_{x} K_{z}$ and $\nabla_{y} K_{z}$, along with the Hölder estimates as done in Corollary 2.12.

The decay estimates obtained in Theorem 3.1 can now we used to transfer the semigroup $e^{-z \mathscr{L}}$, originally defined on $L^{2}\left(\mathrm{R}^{n}\right)$, to $L^{p}\left(\mathrm{R}^{n}\right), 1 \leq p \leq \infty$. Indeed let

$$
e^{-z \mathscr{L}} u(x)=\int_{\mathrm{R}^{n}} K_{z}(x, y) \frac{1}{b}(y) u(y) d y .
$$

COROllary 3.2. Given $\mu>\omega$, there exists $C=C(\mu, \omega)$ such that for any $z \in S_{\left(\frac{\pi}{2}-\mu\right)+}^{0}$,

$$
\begin{gathered}
\left\|e^{-z \mathscr{L}}\right\|_{p, p}+|z|^{\frac{1}{2}} \sum_{j}\left\|\frac{\partial}{\partial x_{j}} e^{-z \mathscr{L}}\right\|_{p, p}+|z|^{\frac{1}{2}} \sum_{j}\left\|e^{-z \mathscr{L}} b \frac{\partial}{\partial x_{j}}\right\|_{p, p} \leq C \\
\text { for all } 1 \leq p \leq \infty .
\end{gathered}
$$

In particular, $-\mathscr{L}$ generates a strongly continuous holomorphic semigroup in $L^{p}\left(\mathrm{R}^{n}\right)$ when $1 \leq p<\infty$.

Proof. Apply Theorem 3.1 with $M>n$ to obtain for any $1 \leq p \leq \infty$ the uniform bound

$$
\left\|e^{-z \mathscr{L}}\right\|_{p, p} \leq \frac{C^{\prime}}{|z|^{\frac{n}{2}}} \int_{\mathrm{R}^{n}} \frac{1}{\left(1+\frac{|x-y|}{\sqrt{|z|}}\right)^{M}} d y \leq C .
$$

The derivative estimates are proved similarly.

REMARK. A consequence of this result is the following statement about regularity of solutions to the following parabolic equation. Given $u \in L^{2}\left(\mathrm{R}^{n}\right)$ let $U(x, t)$ be the solution of 


$$
\begin{cases}\frac{\partial U}{\partial t}(x, t)=b(x) \Delta U(x, t) & \text { for } x \in \mathrm{R}^{n}, t>0 \\ U(x, 0)=u(x) & \text { for } x \in \mathrm{R}^{n}\end{cases}
$$

given by $U(x, t)=e^{-t \mathscr{L}} u(x)$. Then there exists $C>0$ such that, for all $t>0$ and $1<p<\infty$,

$$
\|U(., t)\|_{p}+\sqrt{t}\|\nabla U(., t)\|_{p} \leq C\|u\|_{p} .
$$

A further regularity result is presented in Section 5(iii).

We now improve Proposition 1.1 by showing that $\mathscr{L}$ is type $\omega$ in $L^{p}\left(\mathrm{R}^{n}\right)$ for all $p$. We also obtain bounds on the Green's functions.

Theorem 3.3. The operator $\mathscr{L}$ is of type $\omega$ in $L^{p}\left(\mathrm{R}^{n}\right)$ for all $1 \leq p \leq \infty$. Let $g_{\lambda}(x, y)$ denote the distributional kernel of $(\mathscr{L}-\lambda I)^{-1} b$ with $\lambda \notin S_{\omega+}^{0}$. Then, for any $\mu>\omega$ and $M \geq 1$ there exists a positive constant $c=c_{\mu, M}$ such that

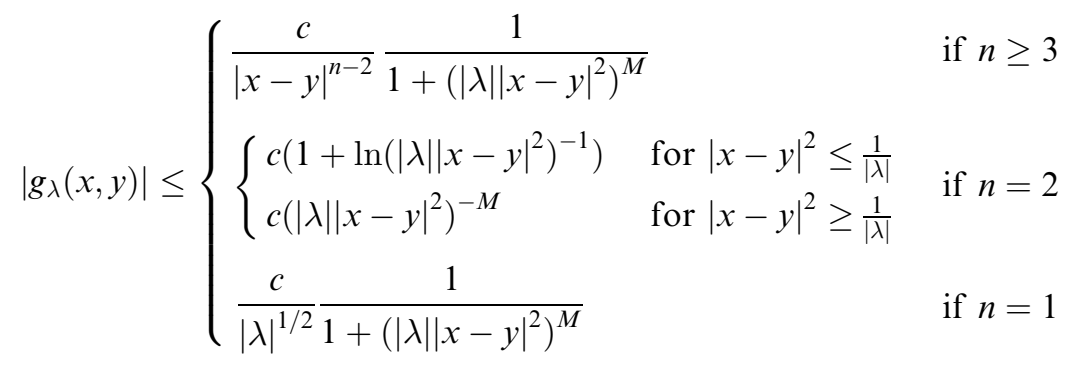

$$
\begin{aligned}
& \left|\nabla_{x, y} g_{\lambda}(x, y)\right| \leq \begin{cases}\frac{c}{|x-y|^{n-1}} \frac{1}{1+\left(|\lambda||x-y|^{2}\right)^{M}} & \text { if } n \geq 2 \\
\begin{cases}c\left(1+\ln \left(|\lambda||x-y|^{2}\right)^{-1}\right) & \text { for }|x-y|^{2} \leq \frac{1}{|\lambda|} \\
c\left(|\lambda||x-y|^{2}\right)^{-M} & \text { for }|x-y|^{2} \geq \frac{1}{|\lambda|}\end{cases} & \text { if } n=1\end{cases}
\end{aligned}
$$

Proof. The operator $\mathscr{L}$ is of type $\omega$ in $L^{p}\left(\mathrm{R}^{n}\right)$ because $-\mathscr{L}$ is the generator of a holomorphic semigroup [28]. This is because

$$
(\mathscr{L}-\lambda I)^{-1}=\int_{\gamma} e^{z \lambda} e^{-z \mathscr{L}} d z,
$$

where $\gamma$ is the ray $\left\{r e^{i \theta}: 0<r<\infty\right\}$ with $\theta$ chosen to satisfy $\frac{\pi}{2}-\frac{\mu+\omega}{2}<\theta<\frac{\pi}{2}-\omega \quad$ if $\quad \mu<\arg \lambda \leq \pi \quad$ and $\quad-\frac{\pi}{2}+\frac{\mu+\omega}{2}>\theta>-\frac{\pi}{2}+\omega \quad$ if $-\mu>\arg \lambda>-\pi$. With this choice of $\theta$ we have $|\arg (z \lambda)|>\frac{\pi}{2}+\frac{\mu-\omega}{2}$ for all $\lambda \notin S_{\mu+}^{0}, z \in \gamma$.

Let $-\beta=\cos (\arg (z \lambda))$. Thus Corollary 3.2 implies for $\lambda \notin S_{\mu+}^{0}$ that 


$$
\left\|(\mathscr{L}-\lambda I)^{-1}\right\|_{p, p} \leq c_{\mu} \int_{0}^{\infty} e^{-\beta|\lambda| r} d r \leq \frac{c_{\mu}}{|\lambda|} .
$$

By Theorem 3.1 we have that for any $M^{\prime} \geq 1$,

$$
\left|g_{\lambda}(x, y)\right|=\left|\int_{\gamma} e^{z \lambda} K_{z}(x, y) d z\right| \leq c \int_{0}^{\infty} e^{-\beta|\lambda| r} \frac{1}{r^{\frac{n}{2}}} \frac{1}{1+\left(\frac{|x-y|}{\sqrt{r}}\right)^{M^{\prime}}} d r .
$$

Let us first consider $n \geq 2$. Then, we can bound

$$
\begin{aligned}
\left|g_{\lambda}(x, y)\right| & \leq c \int_{0}^{\infty} e^{-\beta|\lambda| r} \frac{1}{r^{\frac{n}{2}}} \frac{1}{1+\left(\frac{|x-y|}{\sqrt{r}}\right)^{M^{\prime}}} d r \\
& \leq c \int_{0}^{\infty} e^{-\beta|\lambda| r} r^{-n / 2} r^{M^{\prime} / 2} d r|x-y|^{-M^{\prime}} \\
& =\frac{c}{|x-y|^{n-2}} \frac{1}{\left(|\lambda|^{\frac{1}{2}}|x-y|\right)^{2 M}}
\end{aligned}
$$

on taking $M^{\prime}=2 M+n-2$. On the other hand, if $n \geq 3$ we also have that

$$
\left|g_{\lambda}(x, y)\right| \leq c \int_{0}^{|x-y|^{2}} r^{\frac{M^{\prime}-n}{2}} d r|x-y|^{-M^{\prime}}+c \int_{|x-y|^{2}}^{\infty} r^{-\frac{n}{2}} d r \leq c|x-y|^{-n+2},
$$

so that we have obtained the required bound on $\left|g_{\lambda}(x, y)\right|$ when $n \geq 3$.

If $n=2$ we improve the first bound when $|x-y|^{2}<\frac{1}{|\lambda|}$ as follows,

$$
\begin{aligned}
\left|g_{\lambda}(x, y)\right| & \leq c \int_{0}^{|x-y|^{2}} r^{\frac{M^{\prime}}{2}-1} d r|x-y|^{-M^{\prime}}+c \int_{|x-y|^{2}}^{\infty} e^{-\beta|\lambda| r} r^{-1} d r \\
& \leq c+c \int_{|x-y|^{2}}^{\frac{1}{\lambda \mid}} r^{-1} d r+c \int_{\frac{1}{|\lambda|}}^{\infty} e^{-\beta|\lambda| r} r^{-1} d r \\
& \leq c+c \ln \left(|\lambda||x-y|^{2}\right)^{-1}+c .
\end{aligned}
$$

The bounds on the gradient of $g_{\lambda}(x, y)$ for $n \geq 2$ follow easily from the gradient bounds for $K_{z}(x, y)$ obtained in Theorem 3.1. Indeed we have that

$$
\left|\nabla_{x, y} g_{\lambda}(x, y)\right| \leq \frac{c}{|x-y|^{n-1}} \frac{1}{\left(|\lambda|^{\frac{1}{2}}|x-y|\right)^{2 M}} .
$$

On the other hand we also have that

$$
\left|\nabla_{x, y} g_{\lambda}(x, y)\right| \leq c \int_{0}^{|x-y|^{2}} r^{\frac{M^{\prime}-(n+1)}{2}} d r|x-y|^{-M^{\prime}}+c \int_{|x-y|^{2}}^{\infty} r^{-\frac{n+1}{2}} d r \leq c|x-y|^{-n+1} .
$$

This completes the proof of all the bounds when $n \geq 2$.

We leave to the reader to verify the estimates in the one dimensional case. 
Corollary 3.4. Proposition 1.2 (1) can be extended to hold for any $\lambda \notin S_{\omega+}$. Thus $(\mathscr{L}-\lambda I)^{-1}: L^{p} \rightarrow W^{1, p}$ for $1 \leq p \leq \infty$ and all $\lambda \notin S_{\omega+}$.

Proof. The proof is straightforward from the estimates obtained in Theorem 3.3.

\section{\$4. Quadratic estimates and functional calculi of $-b \Delta$}

In this section we make use of the kernel estimates obtained in $\S 2$ to prove that $\mathscr{L}$ and $\mathscr{L}^{\prime}$ satisfy quadratic estimates. It then follows from [30] that $\mathscr{L}$ has a bounded $H^{\infty}\left(S_{\mu+}^{0}\right)$ functional calculus in $L^{2}\left(\mathrm{R}^{n}\right)$ for all $\mu>\omega$. Further, on applying Calderon-Zygmund theory, it follows that $\mathscr{L}$ has a bounded $H^{\infty}\left(S_{\mu+}^{0}\right)$ functional calculus in $L^{p}\left(\mathrm{R}^{n}\right), 1<p<\infty$.

Let $\psi_{t}(z)=\psi(t z)$ with $\psi \in \Psi\left(S_{\mu+}^{0}\right)$ and let $\delta$ be such that $\omega<\delta<\mu$. Let $\Gamma$ be an unbounded contour consisting of two rays in $\mathrm{C}$ given by $\{\zeta \in \mathrm{C}:|\arg \zeta|=\delta\}$. Define

$$
Q_{t} u=\psi_{t}(\mathscr{L}) b u=\frac{1}{2 \pi i} \int_{\Gamma} \psi(\zeta)(t \mathscr{L}-\zeta I)^{-1} b u d \zeta .
$$

We say that $\mathscr{L}$ satisfies quadratic estimates if

$$
\|u\|_{\mathscr{L}}=\left\{\int_{0}^{\infty}\left\|\psi_{t}(\mathscr{L}) u\right\|_{2}^{2} \frac{d t}{t}\right\}^{\frac{1}{2}} \leq c\|u\|_{2}, \quad u \in L^{2}\left(\mathrm{R}^{n}\right)
$$

where $\psi_{t}(\mathscr{L})=\psi(t \mathscr{L})$ for some $\psi \in \Psi\left(S_{\mu+}^{0}\right)$. Recall that different choices of $\mu>\omega$ and of $\psi \in \Psi\left(S_{\mu+}^{0}\right)$ lead to equivalent quadratic norms $\|u\|_{\mathscr{L}}[32,1]$.

Proposition 4.1. Let $N \geq 1+\frac{n}{4}$ and let $\psi \in \Psi\left(S_{\mu+}^{0}\right)$ be such that

$$
|\psi(\zeta)| \leq \frac{C|\zeta|^{k}}{1+|\zeta|^{k+M}}
$$

where $k \geq 1$ and $\frac{M}{2}>k+\frac{n}{2}$ satisfies,

$$
\int_{0}^{\infty} \xi^{j} \psi(\xi) d \xi=0 \quad \text { for } 0 \leq j \leq(2 N-1) .
$$

Let $q_{t}(x, y)$ denote the kernel of $Q_{t}=\psi_{t}(\mathscr{L}) b$. Then there exist positive constants $M=M(n, k)$ and $c=c\left(\mu, \omega,\|b\|_{\infty},\left\|\frac{1}{b}\right\|_{\infty}, M\right)$ such that

$$
\left|q_{t}(x, y)\right|+t^{\frac{1}{2}}\left|\nabla_{x} q_{t}(x, y)\right|+t^{\frac{1}{2}}\left|\nabla_{y} q_{t}(x, y)\right| \leq \frac{c}{t^{\frac{n}{2}}} \frac{1}{\left(1+\frac{|x-y|^{2}}{t}\right)^{M}}
$$

Proof. Because of the cancellation $\int_{0}^{\infty} \xi^{j} \psi(\xi) d \xi=0$, there exists a function $\underline{\psi} \in \Psi\left(S_{\mu+}^{0}\right)$ such that $\underline{\psi}^{(2 N-1)}=\psi$. Actually, $|\underline{\psi}(\zeta)| \leq c \frac{|\zeta|^{k+2 N-1}}{1+|\zeta|^{k+M}}$. Then, 


$$
\begin{aligned}
Q_{t} u=\psi_{t}(\mathscr{L}) b u & =\frac{1}{2 \pi i} \int_{\Gamma} \psi(\zeta)(t \mathscr{L}-\zeta I)^{-1} b u d \zeta \\
& =\frac{(2 N-1) !}{2 \pi i} \int_{\Gamma} \psi(\zeta)(t \mathscr{L}-\zeta I)^{-2 N} b u d \zeta
\end{aligned}
$$

so the kernel $q_{t}(x, y)$ of $Q_{t}$ satisfies

$$
q_{t}(x, y)=\frac{c}{t^{2 N}} \int_{\Gamma} \underline{\psi}(\zeta) G_{\frac{\zeta}{t}}(x, y) d \zeta
$$

where $G_{\frac{\zeta}{t}}(x, y)$ is the kernel of $\left(\mathscr{L}-\frac{\zeta}{t} I\right)^{-2 N}$. By Theorem 2.1,

$$
\left|G_{\frac{\zeta}{t}}(x, y)\right| \leq c_{\mu} \frac{|\zeta|^{\frac{n}{2}-2 N}}{t^{\frac{n}{2}-2 N}} \frac{1}{\left(1+\left|\frac{\zeta}{t}\right|^{\frac{1}{2}}|x-y|\right)^{M}}
$$

for any $M>0$. In particular taking $M$ so that $\frac{M}{2}>k+\frac{n}{2}$,

$$
\begin{aligned}
\left|q_{t}(x, y)\right| & \leq \frac{c_{\mu}}{t^{\frac{n}{2}}} \int_{\Gamma}|\underline{\psi}(\zeta)| \frac{|\zeta|^{\frac{n}{2}-2 N}}{\left(1+\left|\frac{\zeta}{t}\right|^{\frac{1}{2}}|x-y|\right)^{M}}|d \zeta| \\
& \leq \frac{c_{\mu}}{t^{\frac{n}{2}}} \min \left\{1,\left(\frac{t}{|x-y|^{2}}\right)^{k+\frac{n}{2}}\right\} .
\end{aligned}
$$

We have obtained 1 by bounding the whole integrand by $\frac{|\zeta|^{k+\frac{n}{2}-1}}{1+|\zeta|^{k+M}}$ with $M$ chosen as above; in particular $M>\frac{n}{2}$. We have obtained $\left(\frac{t}{|x-y|^{2}}\right)^{k+\frac{n}{2}}$ by bounding the whole integrand by $\frac{|\zeta|^{k+\frac{n}{2}-1}}{1+|\zeta|^{\frac{M}{2}}|x-y|^{M} t^{-\frac{M}{2}}}$, and using $\frac{M}{2}>k+\frac{n}{2}$.

The estimates on the gradient bounds are obtained similarly by integrating the gradient bounds obtained in Theorem 2.1 for $G_{\frac{\zeta}{t}}(x, y)$.

THeorem 4.2. Let $\psi_{t}(\zeta)$ and $Q_{t}$ be as in Proposition 4.1, so that $Q_{t}^{\prime}=b \psi_{t}\left(\mathscr{L}^{\prime}\right)$ where $\mathscr{L}^{\prime}=-\Delta b$. Then there exists a constant $c$ such that,

$\left\{\int_{0}^{\infty}\left\|Q_{t} u\right\|^{2} \frac{d t}{t}\right\}^{1 / 2} \leq c\|u\|$ and $\left\{\int_{0}^{\infty}\left\|Q_{t}^{\prime} v\right\|^{2} \frac{d t}{t}\right\}^{1 / 2} \leq c\|v\|, u, v \in L^{2}\left(\mathrm{R}^{n}\right)$.

We shall prove this result by appealing to the following powerful theorem of S. Semmes [36]. (Semmes result is actually somewhat stronger than this.)

THEOREM [S]. Let $\theta_{\tau}(x, y)$ be complex-valued functions on $\mathbf{R}^{n} \times \mathbf{R}^{n}, \tau>0$, such that for some $C, \delta>0$, and some function $\beta \in L^{\infty}\left(\mathrm{R}^{n}\right)$, satisfying $\operatorname{Re} \beta \geq \delta$, 


$$
\begin{gathered}
\left|\theta_{\tau}(x, y)\right| \leq \frac{C \tau^{2}}{(\tau+|x-y|)^{n+2}} \\
\left|\theta_{\tau}(x, y)-\theta_{\tau}\left(x, y^{\prime}\right)\right| \leq C \frac{\tau\left|y-y^{\prime}\right|}{(\tau+|x-y|)^{n+2}} \\
\int_{\mathrm{R}^{n}} \theta_{\tau}(x, y) \beta(y) d y=0 .
\end{gathered}
$$

Then there exists $c$ such that

$$
\left\{\int_{0}^{\infty}\left\|\int_{\mathrm{R}^{n}} \theta_{\tau}(\cdot, y) u(y) d y\right\|_{2}^{2} \frac{d \tau}{\tau}\right\}^{\frac{1}{2}} \leq c\|u\|_{2}, \quad u \in L_{2}\left(\mathrm{R}^{n}\right) .
$$

Proof of Theorem 4.2. To prove the first estimate, apply Theorem [S] with $\tau^{2}=t$ and $\theta_{\tau}(x, y)=q_{t}(x, y)$, so that (1) and (2) are consequences of Proposition 4.1.

Without any loss of generality let us verify (3) with $\beta=\frac{1}{b}$ in the case $\tau=1$ and thus $t=1$. The decay of $|\psi(\lambda)|$ at zero and infinity imply that $\psi(-b \Delta)(1) \in L^{\infty}$. Moreover, since $(-b \Delta)(1)=0$ we have that for $0<\alpha<1$,

$$
(-b \Delta)^{\alpha}(1)=c_{\alpha} \int_{0}^{\infty} e^{t b \Delta}(-b \Delta) \frac{d t}{t^{\alpha}}(1)=0 .
$$

For $\alpha$ small enough, we can write $\psi(\lambda)=\tilde{\psi}(\lambda) \lambda^{\alpha}$ for some $\tilde{\psi}$ in $\Psi\left(S_{\mu+}^{0}\right)$. Thus $\psi(-b \Delta)(1)=\tilde{\psi}(-b \Delta)(-b \Delta)^{\alpha}(1)=0$, and hence $\int_{\mathrm{R}^{n}} \theta_{1}(x, y) \beta(y) d y=$ $Q_{1}\left(\frac{1}{b}\right)=0$. Therefore

$$
\left\{\int_{0}^{\infty}\left\|Q_{t} u\right\|^{2} \frac{d t}{t}\right\}^{\frac{1}{2}}=\left\{2 \int_{0}^{\infty}\left\|\int_{\mathrm{R}^{n}} \theta_{\tau}(\cdot, y) u(y) d y\right\|_{2}^{2} \frac{d \tau}{\tau}\right\}^{\frac{1}{2}} \leq \sqrt{2} c\|u\|_{2} .
$$

For the dual estimate, apply Theorem [S] with $\theta_{\tau}(x, y)=q_{t}(y, x)$, again proving (3) with $\beta=\frac{1}{b}$, this time starting with $-\Delta b\left(\frac{1}{b}\right)=0$.

Remark. Let $\mathscr{D}=\sum_{j=1}^{n} \frac{\partial}{\partial x_{j}} e_{j}$ denote the Dirac operator acting as a selfadjoint operator in $L^{2}\left(\mathrm{R}^{n}, \mathrm{C}_{(n)}\right)$ where $\mathrm{R}^{n}$ is embedded in the complex Clifford algebra $\mathrm{C}_{(n)}$. Then $\mathscr{D}^{2}=-\Delta$. It is proved in [5] by using complex interpolation, that quadratic estimates for $b \mathscr{D}$ are equivalent to those for $b \mathscr{D}^{2}=-b \Delta$. Quadratic estimates for $b \mathscr{D}$ were previously obtained in [31] via multilinear expansions. This provides an alternative method for deriving Theorem 4.2. Actually [31] was concerned with quadratic estimates for $\left(1-\sum b_{j} e_{j}\right)^{-1} \mathscr{D}$, but the same multilinear estimates apply.

Corollary 4.3. The operator $\mathscr{L}$ has a bounded $H^{\infty}\left(S_{\mu+}^{0}\right)$ functional cal- 
culus in $L^{2}\left(\mathrm{R}^{n}\right)$ for all $\mu>\omega$. That is, for any $\mu>\omega$ there exists a positive constant $c_{\mu}$ such that

$$
\|f(\mathscr{L}) u\|_{2} \leq c_{\mu}\|f\|_{\infty}\|u\|_{2}
$$

for all $u \in L^{2}\left(\mathrm{R}^{n}\right)$ and all $f \in H^{\infty}\left(S_{\mu+}^{0}\right)$.

This result is a consequence of the quadratic estimates for $\mathscr{L}$ and $\mathscr{L}^{\prime}$ [30]. We now generalize it to an $L^{p}$ result by using the kernel estimates and Calderón-Zygmund theory as presented (see e.g. [14]). Such an approach was used previously in proving that elliptic operators in divergence form have bounded $H^{\infty}$ functional calculi in $L^{p}(\Omega)[22,23]$.

THeorem 4.4. If $1<p<\infty$ the operator $\mathscr{L}$ is of type $\omega$ in $L^{p}\left(\mathrm{R}^{n}\right)$ for all $\mu>\omega$. Moreover $\mathscr{L}$ has a bounded $H^{\infty}\left(S_{\mu+}^{0}\right)$ functional calculus in $L^{p}\left(\mathrm{R}^{n}\right)$. That is, for any $\mu>\omega$, there exists a positive constant $c=c(\mu, p)$ such that

$$
\|f(\mathscr{L}) u\|_{p} \leq c\|f\|_{\infty}\|u\|_{p}
$$

for all $u \in L^{2} \cap L^{p}\left(\mathrm{R}^{n}\right)$ and all $f \in H^{\infty}\left(S_{\mu+}^{0}\right)$.

Proof. Let $\mu>\omega$ and $\Gamma$ be as above. Let $\psi$ be any function in $\Psi\left(S_{\mu+}^{0}\right)$. By Corollary 4.3 we know that $\psi(\mathscr{L})$ defines a bounded operator on $L^{2}$ and that $\|\psi(\mathscr{L})\| \leq c_{\mu}\|\psi\|_{\infty}$. For $u \in L^{2} \cap L^{p}\left(\mathbf{R}^{n}\right)$ we write

$$
\psi(\mathscr{L}) b u=\frac{1}{2 \pi i} \int_{\Gamma} \psi(\lambda)(\mathscr{L}-\lambda I)^{-1} b u d \lambda .
$$

We claim now that the kernel estimates for the resolvent obtained in Theorem 3.3 imply that $\psi(\mathscr{L}) b$ has a kernel $k_{\psi}$ satisfying Calderón-Zygmund estimates,

$$
\left|k_{\psi}(x, y)\right|+|x-y|\left|\nabla_{x, y} k_{\psi}(x, y)\right| \leq \frac{c_{\mu}}{|x-y|^{n}}\|\psi\|_{\infty} .
$$

Indeed, let $k_{\psi}(x, y)=\frac{1}{2 \pi i} \int_{\Gamma} \psi(\lambda) g_{\lambda}(x, y) d \lambda$ then we have

$$
\left|k_{\psi}(x, y)\right| \leq \frac{1}{2 \pi} \int_{\Gamma}\|\psi\|_{\infty}\left|g_{\lambda}(x, y)\right||d \lambda| .
$$

The Calderón-Zygmund estimates are now straightforward from the estimates in Theorem 3.3. For example, if $n \geq 3$, then, on taking $M \geq 2$,

$$
\left|k_{\psi}(x, y)\right| \leq \frac{c_{\mu, M}\|\psi\|_{\infty}}{|x-y|^{n-2}} \int_{0}^{\infty} \frac{1}{1+\left(r|x-y|^{2}\right)^{M}} d r \leq \frac{c_{\mu}}{|x-y|^{n}}\|\psi\|_{\infty} .
$$

The estimates for $n=1,2$ and on the derivatives are obtained similarly. 
Therefore we have, by Calderón-Zygmund theory, that $\psi(\mathscr{L}) b$, and thus $\psi(\mathscr{L})$, extends to a bounded operator on $L^{p}$ for all $1<p<\infty$ satisfying

$$
\|\psi(\mathscr{L}) u\|_{p} \leq c_{\mu, p}\|\psi\|_{\infty}\|u\|_{p} .
$$

Since this holds for any $\psi \in \Psi\left(S_{\mu+}^{0}\right)$, by taking limits in the usual way (as explained, for example in [1]) we find that

$$
\|f(\mathscr{L}) u\|_{p} \leq c_{\mu, p}\|f\|_{\infty}\|u\|_{p}
$$

for all $f \in H^{\infty}\left(S_{\mu+}^{0}\right)$ and all $u \in L^{2} \cap L^{p}\left(\mathrm{R}^{n}\right)$.

Remark. Instead of relying on Proposition 4.1 and Corollary 4.3 we could have proved Theorem 4.4 using the $T(b)$-theorem of David, Journé and Semmes.

Let us conclude this section with some comments about quadratic estimates for $\mathscr{L}$ in $L^{2}\left(\mathrm{R}^{n}\right)$. First we remark that the quadratic estimates for $\mathscr{L}^{\prime}$ imply reverse quadratic estimates for $\mathscr{L}[32,1]$ so that actually

$$
\|u\|_{\mathscr{L}}=\left\{\int_{0}^{\infty}\left\|\psi_{t}(\mathscr{L}) u\right\|^{2} \frac{d t}{t}\right\}^{\frac{1}{2}} \approx\|u\|_{2}
$$

for all $u \in L^{2}\left(\mathrm{R}^{n}\right)$. A more conventional way of viewing this is as follows.

Given $u \in L^{2}\left(\mathrm{R}^{n}\right)$ let $U(x, t)$ be the solution of

$$
\begin{cases}\frac{\partial U}{\partial t}(x, t)=b(x) \Delta U(x, t) & \text { for } x \in \mathrm{R}^{n}, t>0, \\ U(x, 0)=u(x) & \text { for } x \in \mathrm{R}^{n}\end{cases}
$$

given by $U(x, t)=e^{t b \Delta} u(x)$. If we choose $\psi$ to be the function given by $\psi(\zeta)=\zeta^{k} e^{-\zeta}$ for some integer $k \geq 1$, then

$$
\psi_{t}(-b \Delta) u(x)=t^{k}(-b \Delta)^{k} e^{t b \Delta} u(x)=t^{k}(-b \Delta)^{k} U(x, t)=(-t)^{k} \frac{\partial^{k} U}{\partial t^{k}}(x, t) .
$$

Thus the quadratic estimates we have proved in this section become

$$
\left\{\int_{0}^{\infty} \int_{\mathrm{R}^{n}}\left|\frac{\partial^{k} U}{\partial t^{k}}(x, t)\right|^{2} t^{2 k-1} d x d t\right\}^{\frac{1}{2}} \approx\|u\|_{2} .
$$

They can also be expressed in terms of solutions $V$ of the elliptic equation

$$
\begin{cases}\frac{\partial^{2} V}{\partial t^{2}}(x, t)+b(x) \Delta V(x, t)=0 & \text { for } x \in \mathrm{R}^{n}, t>0, \\ V(x, 0)=u(x) & \text { for } x \in \mathrm{R}^{n}\end{cases}
$$


given by $V(x, t)=e^{-t \sqrt{-b \Delta}} u(x)$. This time we choose $\psi(\zeta)=\zeta e^{-\sqrt{\zeta}}$, so that by setting $t^{2}=\tau$, we have

$$
\psi_{\tau}(-b \Delta) u(x)=-t^{2} b \Delta e^{-t \sqrt{-b \Delta}} u(x)=t^{2} \frac{\partial^{2} V}{\partial t^{2}}(x, t) .
$$

Thus the quadratic estimate

$$
\begin{aligned}
& \left\{\int_{0}^{\infty}\left\|\psi_{\tau}(-b \Delta) u\right\|^{2} \frac{d \tau}{\tau}\right\}^{\frac{1}{2}} \approx\|u\| \quad \text { becomes } \\
& \left\{\int_{0}^{\infty} \int_{\mathrm{R}^{n}}\left|\frac{\partial^{2} V}{\partial t^{2}}(x, t)\right|^{2} t^{3} d x d t\right\} \approx\|u\|_{2} .
\end{aligned}
$$

\section{§5. Extensions and applications}

(i) Generalized Riesz transforms

Let us use the boundedness of the $H^{\infty}$ functional calculus of $\mathscr{L}$ and the kernel bounds to prove that the generalized Riesz transforms $R_{j}=\frac{\partial}{\partial x_{j}} \mathscr{L}^{-\frac{1}{2}}$ are bounded operators on $L^{p}\left(\mathrm{R}^{n}\right)$. Here $\mathscr{L}^{-\frac{1}{2}}=(\sqrt{\mathscr{L}})^{-1}$ where $\sqrt{\mathscr{L}}$ is the unique operator of type $\frac{\omega}{2}$ whose square is $\mathscr{L}$. Recall that, since $\sqrt{\mathscr{L}}$ is a one-one operator of type $\frac{\omega}{2}$ in $L^{p}\left(\mathbf{R}^{n}\right)$, then $\mathscr{D}\left(\mathscr{L}^{-\frac{1}{2}}\right)=\mathscr{R}(\sqrt{\mathscr{L}})$ is dense in $L^{p}\left(\mathrm{R}^{n}\right)$. These observations are due to Duong.

THEOREM 5.1. The operators $\frac{\partial}{\partial x_{j}} \mathscr{L}^{-\frac{1}{2}}, j=1, \ldots, n$, are bounded operators with dense domain $\mathscr{D}\left(\mathscr{L}^{-\frac{1}{2}}\right)$ in $L^{p}\left(\mathrm{R}^{n}\right)$, and hence have unique extensions to bounded operators $R_{j}$ on $L^{p}\left(\mathrm{R}^{n}\right), 1<p<\infty$.

Proof. First consider the case $p=2$. Since $\mathscr{L}$ has a bounded $H^{\infty}$ functional calculus in $L^{2}\left(\mathrm{R}^{n}\right)$, its fractional powers interpolate by the complex method $[40,30,5]$. Thus $\mathscr{D}(\sqrt{\mathscr{L}})=\left[\mathscr{D}(\mathscr{L}), L^{2}\right]_{\frac{1}{2}}=\left[W^{2,2}, L^{2}\right]_{\frac{1}{2}}=W^{1,2}$ with $\|\sqrt{\mathscr{L}} u\| \approx\|\nabla u\|$ for all $u \in W^{1,2}$. Here [ , $]_{\frac{1}{2}}$ is the half way complex interpolation space. See, e.g., [11]. Therefore we have that for all $v \in \mathscr{D}\left(\mathscr{L}^{-\frac{1}{2}}\right)=\mathscr{R}(\sqrt{\mathscr{L}}),\|v\|_{2}^{2} \approx \sum_{j=1}^{n}\left\|\frac{\partial}{\partial x_{i}} \mathscr{L}^{-\frac{1}{2}} v\right\|_{2}^{2}$ from which it follows that $\frac{\partial}{\partial x_{j}} \mathscr{L}^{-\frac{1}{2}}$ has a bounded extension to $L^{2}\left(\mathrm{R}^{n}\right)$.

In order to extend this result to the case when $1<p<\infty$, write

$$
\mathscr{L}^{-\frac{1}{2}} u=c \int_{0}^{\infty}(\mathscr{L}+\tau)^{-2 N} u \tau^{2 N-\frac{3}{2}} d \tau, \quad u \in \mathscr{R}(\sqrt{\mathscr{L}}),
$$

where $N \geq 1+\frac{n}{4}$ and $\frac{1}{c}=\int_{0}^{\infty}(1+\tau)^{-2 N} \tau^{2 N-\frac{3}{2}} d \tau$. This formula is a consequence of the holomorphic functional calculus for $\mathscr{L}$. Therefore, as bounded operators on $L^{2}\left(\mathrm{R}^{n}\right)$, 


$$
R_{j} b=c \int_{0}^{\infty} \frac{\partial}{\partial x_{j}}(\mathscr{L}+\tau)^{-2 N} b \tau^{2 N-\frac{3}{2}} d \tau
$$

By Theorem 2.1, the kernel $k(x, y)$ of $\frac{\partial}{\partial x_{j}} \mathscr{L}^{-\frac{1}{2}} b$, which is given by

$$
k(x, y)=c \int_{0}^{\infty} \frac{\partial}{\partial x_{j}} G_{\tau}(x, y) \tau^{2 N-\frac{3}{2}} d \tau,
$$

satisfies Calderón-Zygmund bounds

$$
\begin{aligned}
|k(x, y)| & \leq c \int_{0}^{\infty} \frac{\tau^{\frac{n+1}{2}-2 N}}{1+\left(\tau^{1 / 2}|x-y|\right)^{M}} \tau^{2 N-\frac{3}{2}} d \tau \\
& =c|x-y|^{-n} \int_{0}^{\infty} \frac{s^{n / 2}}{1+s^{M / 2}} \frac{d s}{s}=c|x-y|^{-n},
\end{aligned}
$$

together with Hölder bounds for any $\eta, 0<\eta<1$. This fact, together with the $L^{2}$ result, implies that $R_{j}$ is bounded on $L^{p}\left(\mathrm{R}^{n}\right)$ for $1<p<\infty$.

(ii) The square root problem for $\Delta b \Delta$

Let $T$ denote the operator in $L^{2}\left(\mathrm{R}^{n}\right)$ associated with the closed sectorial sesquilinear form $\int_{\mathrm{R}^{n}} b \Delta u \Delta v$ with domain $W^{2,2}\left(\mathrm{R}^{n}\right)$ [28]. Then $T=\Delta b \Delta$ with domain $\mathscr{D}(T)=\left\{u \in W^{2,2}\left(\mathrm{R}^{n}\right): b \Delta u \in W^{2,2}\left(\mathrm{R}^{n}\right)\right\}$. By Theorem VI-2.1 of [28], $T$ is a maximal accretive operator in $L^{2}\left(\mathrm{R}^{n}\right)$. The square root problem asks whether the domain of its unique maximal accretive square root $\sqrt{T}$ equals the domain of the form.

The answer is positive, on account of the quadratic estimates for $\mathscr{L}$ and $\mathscr{L}^{\prime}$, as is seen by applying Theorem 7.1 of [5]. We thus have the following result.

THEOREM 5.2. Let $T=\Delta b \Delta$ be the operator in $L^{2}\left(\mathrm{R}^{n}\right)$ with domain $\mathscr{D}(T)=\left\{u \in W^{2,2}\left(\mathrm{R}^{n}\right): b \Delta u \in W^{2,2}\left(\mathrm{R}^{n}\right)\right\}$. Its square root $\sqrt{T}$ has domain $\mathscr{D}(\sqrt{T})=W^{2,2}\left(\mathrm{R}^{n}\right)$ with $\|\sqrt{T} u\|_{2} \approx \sum_{j, k}\left\|\frac{\partial^{2} u}{\partial x_{j} \partial x_{k}}\right\|_{2}$ for all $u \in W^{2,2}\left(\mathrm{R}^{n}\right)$.

Let us give a brief outline of the proof. For any one-one operator $S$, let $\mathscr{D}_{S}$ denote the completion of $\mathscr{D}(S)$, the domain of $S$, under the norm $\|S u\|$.

Since, by Corollary $4.3, \mathscr{L}$ and hence $\mathscr{L}^{2}$ have bounded $H^{\infty}$ functional calculi in $\mathscr{L}^{2}\left(\mathrm{R}^{n}\right)$, the complex interpolation space $\left[\mathscr{D}_{\mathscr{L}^{2}}, L^{2}\left(\mathrm{R}^{n}\right)\right]_{\frac{1}{2}}=$ $\mathscr{D}_{\mathscr{L}}=\mathscr{D}_{\Delta}$. Moreover $\left[\mathscr{D}_{T}, L^{2}\left(\mathrm{R}^{n}\right)\right]_{\frac{1}{2}}=\mathscr{D}_{\sqrt{T}}$ because $T$, being maximal accretive, also has a bounded $H^{\infty}$ functional calculus. Clearly $\mathscr{D}_{\mathscr{L}^{2}}=\mathscr{D}_{T}$. Therefore

$$
\mathscr{D}_{\sqrt{T}}=\left[\mathscr{D}_{T}, L^{2}\left(\mathrm{R}^{n}\right)\right]_{\frac{1}{2}}=\left[\mathscr{D}_{\mathscr{L}^{2}}, L^{2}\left(\mathrm{R}^{n}\right)\right]_{\frac{1}{2}}=\mathscr{D}_{\Delta}
$$

with $\|\sqrt{T} u\|_{2} \approx\|\Delta u\|_{2} \approx \sum_{j, k}\left\|\frac{\partial^{2} u}{\partial x_{j} \partial x_{k}}\right\|_{2}$ for all $u \in \mathscr{D}(\Delta)=W^{2,2}\left(\mathrm{R}^{n}\right)$.

REMARK. This result was previously known to hold via the method of 
multilinear expansions developed by Coifman, McIntosh and Meyer in [13]. Indeed the one dimensional case is equivalent to the square root problem for $-\frac{d}{d x} b \frac{d}{d x}$ which was proved there. The higher dimensional analogues presented in [31] imply the full result.

(iii) Maximal regularity

One consequence of the fact that $\mathscr{L}$ has a bounded $H_{\infty}$ functional calculus in $L^{p}\left(\mathrm{R}^{n}\right)$ for all $\mu>\omega$, is that the purely imaginary powers $\mathscr{L}^{i s}$ of $\mathscr{L}$ are bounded in $L^{p}\left(\mathrm{R}^{n}\right)$ and

$$
\left\|\mathscr{L}^{i s}\right\|_{p, p} \leq c_{\mu, p} e^{\mu|s|}, s \in \mathrm{R} .
$$

To see this, write $\mathscr{L}^{i s}=f_{s}(\mathscr{L})$ where $f_{s}(\zeta)=\zeta^{i s}$, and apply the bound $\left\|f_{s}\right\|_{\infty}=e^{\mu|s|}$.

Dore and Venni [20] have shown that if an invertible operator in $L^{p}\left(\mathrm{R}^{n}\right)$ satisfies $(*)$ with $1<p<\infty$ and $0 \leq \mu<\frac{\pi}{2}$, then maximal regularity results for associated evolution equations follow. Namely, if $1<q<\infty$, then solutions $u(\cdot, t)$ to

$$
\frac{\partial u}{\partial t}(t)+\mathscr{L} u(t)=f(t), \quad 0<t<T, \quad u(0)=0
$$

satisfy, for some $C=C(T, p, q)$, the $L^{q}\left((0, T), L^{p}\left(\mathrm{R}^{n}\right)\right)$ estimate

$$
\int_{0}^{T}\left\|\frac{\partial u}{\partial t}(t)\right\|_{p}^{q} d t+\int_{0}^{T}\|\mathscr{L} u(t)\|_{p}^{q} d t \leq C \int_{0}^{T}\|f(t)\|_{p}^{q} d t .
$$

This result was generalized by Giga and Sohr [27] to cover the case when $\mathscr{L}^{-1}$ is unbounded. They showed that the constant $C$ is independent of $T$, thus proving estimates which are global in time. See also [34, 33, 2]. This kind of estimate is useful for studying regularity and long time behavior of nonlinear equations of the form $\frac{\partial u}{\partial t}(t)+\mathscr{L} u(t)+N(u)=f(t)$, where the nonlinear term $N$ is subordinate to $\mathscr{L}$ (see [27]). In this way, maximal regularity results can be obtained for solutions $u(\cdot, t)$ to

$$
\frac{\partial u}{\partial t}(t)-b \Delta u(t)=f(t), \quad t>0, \quad u(0)=0 .
$$

(iv) Exponential decay

As remarked after Theorem 2.1, the polynomial decay stated there can be improved to exponential decay in $|x-y|$. However we have not pursued this because polynomial decay is sufficient for proving the bounds on the functional calculus and the regularity results for parabolic equations.

After reading a draft of this manuscript, Duong and Ouhabaz [24] have shown by an alternative method that the heat kernel $k_{t}(x, y)$ satisfies Gaus- 
sian bounds, and extended these results to include heat kernels of other operators of the form $b A$ for which the heat kernel of $A$ itself satisfies Gaussian bounds.

(v) The matrix-valued case

Suppose that $B$ is a matrix-valued function with coefficients in $L^{\infty}\left(\mathrm{R}^{n}, \mathrm{C}\right)$, satisfying

$$
\operatorname{Re} \sum b_{j k}(x) \zeta_{k} \overline{\zeta_{j}} \geq \kappa|\zeta|^{2} \text { for all } \zeta=\left(\zeta_{j}\right) \in \mathrm{C}^{m} \text { and almost all } x \in \mathrm{R}^{n} .
$$

Then for some $\omega<\frac{\pi}{2}$, multiplication by $B$ is an invertible bounded $\omega$-accretive operator in $L^{2}\left(\mathrm{R}^{n}, \mathrm{C}^{m}\right)$.

Define $\mathscr{L}=-B \Delta$ by $\mathscr{L} u_{j}=\sum_{k} b_{j k} \Delta u_{k}$ for all $u \in W^{2,2}\left(\mathrm{R}^{n}, \mathrm{C}^{m}\right)$. Then $\mathscr{L}$ is a one-one operator of type $\omega$ and all our results remain valid when $b$ is replaced by $B$ and $W^{k, p}\left(\mathrm{R}^{n}\right)$ by $W^{k, p}\left(\mathrm{R}^{n}, \mathrm{C}^{m}\right)$. Just repeat the proofs above step by step. All we have used is that $\mathscr{L}$ is of type $\omega$, and that the multiplication operator $B$ is an invertible bounded $\omega$-accretive operator. Note that Theorem $[\mathrm{S}]$ stated in $\S 4$ remains valid when working with functions taking values in some finite dimensional algebra [36].

\section{(vi) Higher order operators}

Another generalization is to consider the operator $\mathscr{L}_{r}=b(-\Delta)^{r}$ defined for any $r>0$. Then $\mathscr{L}_{r}$ is a one-one operator of type $\omega$ in $L^{2}\left(\mathrm{R}^{n}, \mathrm{C}^{m}\right)$ since $b$ is bounded, invertible and $\omega$-accretive and $(-\Delta)^{r}$ is selfadjoint. It is a consequence of Theorem 4.2 and [5, Theorem 3.6] that $b(-\Delta)^{r}$ and its dual satisfy quadratic estimates, and hence that $b(-\Delta)^{r}$ has a bounded $H^{\infty}$ functional calculus in $L^{2}\left(\mathrm{R}^{n}\right)$.

In this case we do not know whether the appropriate bounds hold on the kernels of the iterated resolvents, and hence whether $b(-\Delta)^{r}$ has a bounded $H^{\infty}$ functional calculus in $L^{p}\left(\mathrm{R}^{n}\right)$. The step which may fail is the proof of Proposition 1.1 (2) in which we used sharp bounds on the Bessel potentials in proving that $\left\|(-\Delta+I)^{-1}\right\|_{p, p} \leq 1$. It would be interesting to obtain more general results by finding a way to circumvent this step. Similar comments apply to operators of the form $B \mathscr{D}$ where $\mathscr{D}$ is the Dirac operator and $B$ is an appropriate multiplication operator.

One case in which we can proceed is when the power $r$ is a sufficiently large integer, so that we do not need to iterate the resolvent to get from $L^{2}$ to $L^{\infty}$, but can do so directly without using $L^{p}$ results along the way. In this case the following resolvent bounds can be obtained. Details are left to the reader.

Proposition 5.3. Let $\mathscr{L}_{r}=b(-\Delta)^{r}$ where $r$ is an integer larger than $\frac{1}{2}+\frac{n}{4}$. Then the distributional kernel $G_{\lambda}(x, y)$ of $\left(\mathscr{L}_{r}-\lambda I\right)^{-2} b$ belongs to 
$C^{1}\left(\mathrm{R}^{n} \times \mathrm{R}^{n}\right)$ for all $\lambda \notin S_{\omega+}$. Moreover, given $M \geq 0$ and $\mu>\omega$, there exists a positive constant $C=C\left(M, \mu, \omega,\|b\|_{\infty},\left\|b^{-1}\right\|_{\infty}\right)$ such that for all $\lambda \notin S_{\mu+}$ and for all $x, y \in \mathrm{R}^{n}, x \neq y$,

$$
|\lambda|^{\frac{1}{2 r}}\left|G_{\lambda}(x, y)\right|+\left|\nabla_{x} G_{\lambda}(x, y)\right|+\left|\nabla_{y} G_{\lambda}(x, y)\right| \leq \frac{C|\lambda|^{\frac{n+1}{2 r}-2}}{1+\left(|\lambda|^{\frac{1}{2 r}}|x-y|\right)^{M}} .
$$

As already indicated, the following result now follows.

TheOREm 5.4. If $r$ is an integer larger than $\frac{1}{2}+\frac{n}{4}$ and $1<p<\infty$, then the operator $\mathscr{L}_{r}=b(-\Delta)^{r}$ is of type $\omega$ and has a bounded $H^{\infty}\left(S_{\mu+}^{0}\right)$ functional calculus in $L^{p}\left(\mathrm{R}^{n}\right)$ for all $\mu>\omega$. That is, there exists $c=c(\mu, p)$ such that

$$
\left\|f\left(\mathscr{L}_{r}\right) u\right\|_{p} \leq c\|f\|_{\infty}\|u\|_{p}
$$

for all $u \in L^{2} \cap L^{p}\left(\mathrm{R}^{n}\right)$ and all $f \in H^{\infty}\left(S_{\mu+}^{0}\right)$.

Remark. In Proposition 5.3, Hölder bounds on $\nabla G_{\lambda}$ can also be obtained.

Remark. As noted above it would be interesting to know whether analogues of the preceding two results hold for all $r>0$ (with the power -2 in Proposition 5.3 replaced by $-2 N$ ), and also for operators of the form $B \mathscr{D}^{s}$ where $\mathscr{D}$ is the Dirac operator and $B$ is an appropriate multiplication operator, especially when $s=1$.

(vii) Lower order terms

The results about functional calculi remain valid when lower order terms are added to the operator $\mathscr{L}$. In particular, the following results hold.

Theorem 5.5. (1) Let $\beta_{j}, \beta \in L^{\infty}\left(\mathrm{R}^{n}\right)$, and suppose $\nu>\omega$ and $1<p<\infty$. Then there exists $C(\nu, p)$ such that $-b \Delta+\sum_{j=1}^{n} \beta_{j} \frac{\partial}{\partial x_{j}}+\beta+C$ is an invertible operator of type $\nu$ in $L^{p}\left(\mathrm{R}^{n}\right)$ for all $C>C(\nu, p)$. These operators have bounded $H^{\infty}\left(S_{\mu+}^{0}\right)$ functional calculi in $L^{p}\left(\mathrm{R}^{n}\right)$ for all $\mu>\nu$.

(2) The operator $b(-\Delta+I)$ has a bounded $H^{\infty}$ functional calculus in $L^{2}\left(\mathrm{R}^{n}\right)$.

Both parts of this theorem follow directly Theorem 4.4 and the following result, on taking $S=\mathscr{L}, T$ equal to the perturbed operator, and $\alpha=\frac{1}{2}$.

Proposition 5.6. Let $S$ and $T$ be two one-one operators of type $\nu$ in a $\mathrm{Ba}$ nach space $\mathscr{X}$, each with dense domain and dense range, and let $\nu<\mu<\pi$ and $0<\alpha<1$. Suppose

(i) $S$ has a bounded $H^{\infty}\left(S_{\mu+}^{0}\right)$ functional calculus in $\mathscr{X}$;

(ii) $T=S+P$ where $\mathscr{D}(P) \supset \mathscr{D}\left(S^{\alpha}\right)$, and $\|P u\| \leq c\left\|S^{\alpha} u\right\|$ for some $c>0$ and for all $u \in \mathscr{D}\left(S^{\alpha}\right)$; 
(iii) $T$ is invertible.

Then $T$ has a bounded $H^{\infty}\left(S_{\mu+}^{0}\right)$ functional calculus in $\mathscr{X}$.

This result was essentially proved by Duong [22], building on a prior result of Dore and Venni for operators with bounded imaginary powers [21].

Remark. Theorem 5.5(2) is also a consequence of Corollary 4.3 and [5, Prop. 8.2] where it is proved that quadratic estimates for $b(-\Delta+I)$ follow from those for $-b \Delta$.

\section{(viii) Bounded domains}

We conclude by indicating that the $L^{2}$ results carry over to operators defined by boundary conditions on domains in $\mathrm{R}^{n}$. We have not considered the kernel bounds and $L^{p}$ theory in this context. This may be an interesting topic for subsequent investigation.

In the following result, $\Omega$ denotes a bounded Lipschitz domain in $\mathrm{R}^{n}$, meaning that the boundary of $\Omega$ can be represented locally by the graph of a Lipschitz function of $(n-1)$ variables. Such a domain has the extension property for Sobolev spaces [38], and also has the property that $\left\|\chi_{\Omega} u\right\|_{W^{s, 2}\left(\mathrm{R}^{n}\right)} \leq c\|u\|_{W^{s, 2}\left(\mathrm{R}^{n}\right)}$ for all positive $s<\frac{1}{2}$, where $\chi_{\Omega}$ is the characteristic function of $\Omega$ [39].

Suppose that $\Delta_{\mathscr{r}}$ is the Laplacian defined from a Dirichlet form on $\mathscr{V}$, where $\mathscr{V}$ is a closed subspace of $W^{1,2}(\Omega)$ which contains $C_{0}^{\infty}(\Omega)$. That is, $\Delta_{\mathscr{r}}$ has the largest domain in $\mathscr{V}$ such that $\left\langle\Delta_{\mathscr{r}} u, v\right\rangle=$ $-\int_{\mathrm{R}^{n}} \sum_{j} \frac{\partial u}{\partial x_{j}}(x) \frac{\partial v}{\partial x_{j}}(x) d x$ for all $v \in \mathscr{V}$. If $\mathscr{V}$ is the closure of $C_{0}^{\infty}(\Omega)$, then $\Delta_{\mathscr{V}}$ is the Dirichlet operator, while if $\mathscr{V}=W^{1,2}(\Omega)$, then $\Delta_{\mathscr{V}}$ is the Neumann operator.

Theorem 5.7. The operator $b\left(-\Delta_{\mathscr{r}}+I\right)$ has a bounded $H^{\infty}$ functional calculus in $L^{2}(\Omega)$.

Proof. The theorem is a consequence of Theorem 5.5(2), [5, Theorem 3.6] and interpolation. It is proved in the same way as results on bounded intervals are derived from results on the whole real line in [6, Theorem 6.4].

This result is stable under the addition of lower order terms as in Theorem 5.5(1).

\section{REFERENCES}

1. D. Albrecht, X.T. Duong and A. McIntosh, Operator theory and harmonic analysis, Workshop in Analysis and Geometry 1995, Part III, Proc. of the CMA, ANU, Canberra (1996), 77-136. 
2. D. Albrecht, E. Franks and A. McIntosh, Holomorphic functional calculi of commuting operators, submitted.

3. H. Amann, M. Hieber and G. Simonett, Bounded $H^{\infty}$-calculus for elliptic operators, Differential Integral Equations 7 (1994), 613-653.

4. P. Auscher, Regularity theorems and heat kernels for elliptic operators, J. London Math. Soc. 54 (1996), 284-296.

5. P. Auscher, A. McIntosh and A. Nahmod, Holomorphic functional calculi of operators, quadratic estimates and interpolation, Indiana Univ. Math. Journal 46 (1997), 375-403.

6. P. Auscher, A. McIntosh and A. Nahmod, The square root problem of Kato in one dimension, and first order elliptic systems, Indiana Univ. Math. Journal 46 (1997).

7. P. Auscher, A. McIntosh and Ph. Tchamitchian, Heat kernels of second order complex elliptic operators and applications, J. Funct. Anal. 152 (1998), 22-72.

8. P. Bauman, Equivalence of the Green's functions for diffusion operators in $\mathrm{R}^{n}$ : a counterexample, Proc. Amer. Math. Soc. 91 (1984), 64-68.

9. P. Bauman, Positive solutions of elliptic equations in nondivergence form and their adjoints, Ark. Mat. 22 (1984), 153-173.

10. R. Beals, Asymptotic behavior of the Green's function and spectral function of an elliptic operator, J. Funct.1 Anal. 5 (1970), 484-503.

11. J. Bergh and J. Löfström. Interpolation Spaces. An Introduction, Springer-Verlag 1976.

12. A.P. Calderón, Cauchy integrals on Lipschitz curves and related operators, Proc. Nat. Acad. Sci. U.S.A. 74 (1977), 1324-1327.

13. R.R. Coifman, A. McIntosh and Y. Meyer, L'intégrale de Cauchy définit un opérateur borné sur $L^{2}$ pour les courbes lipschitziennes, Ann. of Math. 116 (1982), 361-387.

14. R.R. Coifman and Y. Meyer, Au-delà des opérateurs pseudo-difféerentiels, Astérisque 57, Société Math. de France 1978.

15. M. Cowling, I. Doust, A. McIntosh and A. Yagi, Banach space operators with a bounded $H^{\infty}$ functional calculus, J. Austral. Math. Soc. Ser. A 60 (1996), 51-89.

16. G. David and J.L. Journé, A boundedness criterion for generalized Calderón-Zygmund operators, Ann. of Math. 120 (1984), 371-397.

17. G. David, J.L. Journé and S. Semmes, Opérateurs de Calderón-Zygmund, fonctions paraaccrétives et interpolation, Rev. Mat. Iberoamericana 1 (1985), 1-56.

18. E.B. Davies, Heat Kernels and Spectral Theory, Cambridge University Press 1992.

19. E.B. Davies, Uniformly elliptic operators with measurable coefficients, J. Funct. Anal. 132 (1995), 141-169.

20. G. Dore and A. Venni, On the closedness of the sum of two closed operators, Math. Z. 196 (1987), 189-201.

21. G. Dore and A. Venni, Some results about complex powers of closed operators, J. Math. Anal. Appl. 149 (1990), 124-136.

22. X. T. Duong , $H_{\infty}$ functional calculus of elliptic partial differential operators in $L_{p}$ spaces (1990) PhD thesis, Macquarie University.

23. X.T. Duong and A. McIntosh, Functional calculi of second order elliptic partial differential with bounded measurable coefficients, J. Geom. Anal. 6 (1996), 181-205.

24. X.T. Duong and E.M Ouhabaz, Complex multiplicative perturbations of elliptic operators: heat kernel bounds and holomorphic functional calculus, submitted.

25. X.T. Duong and G. Simonett, $H^{\infty}$-calculus for elliptic operators with non-smooth coefficients, Differential Integral Equations, to appear.

26. E.B. Fabes and D.W. Stroock, The $L^{p}$ integrability of Green's functions and fundamental solutions for elliptic and parabolic equations, Duke Math. J. 51 (1984), 997-1016.

27. Y. Giga and H. Sohr, Abstract $L^{p}$ estimates for the Cauchy problem with applications to the Navier-Stokes equations in exterior domains, J. Funct. Anal. 102 (1991), 72-94.

28. T. Kato, Perturbation Theory for Linear Operators, 2nd edition, Springer-Verlag 1976.

29. N. V. Krylov and M.V. Safonov, A certain property of solutions of parabolic equations with measurable coefficients, Math. USSR- Izv. 16 (1981), 151-164. 
30. A. McIntosh, Operators which have an $H^{\infty}$ functional calculus, Miniconference on Operator Theory and Partial Differential Eqs., Proc. of the Centre for Math. Analysis, ANU, Canberra, 14 (1986), 210-231.

31. A. McIntosh, Clifford algebras and the higher dimensional Cauchy integral, Approximation and Function Spaces, Banach Center Publ. 22 (1989), 253-267.

32. A. McIntosh and A. Yagi, Operators of type $\omega$ without a bounded $H_{\infty}$ functional calculus, Miniconference on Operators in Analysis, Proc. of the Centre for Math. Analysis, ANU, Canberra 24 (1989), 159-172.

33. S. Monniaux, A new approach to the Dore-Venni theorem, Math. Nachr., to appear.

34. J. Prüss and H. Sohr, On operators with bounded imaginary powers in Banach spaces, Math. Z. 203 (1990), 429-452.

35. D.W. Robinson, Elliptic Operators and Lie Groups, Oxford University Press, 1991.

36. S. Semmes, Square function estimates and the $T(b)$ theorem, Proc. Amer. Math. Soc. 110 (1990), 721-726.

37. R. Showalter, The Sobolev equation, II, Applicable Anal. 5 (1975), 81-99.

38. E. Stein, Singular Integrals and Differentiability Properties of Functions, Princeton Univ. Press 1970.

39. R. Strichartz, Multipliers on fractional Sobolev spaces, J. Math. Mech. 16 (1967), 1030-1060.

40. A. Yagi, Coincidence entre des espaces d'interpolation et des domaines de puissances fractionnaires d'opérateurs, C.R. Acd. Sci. Paris, Série A 299 (1984), 173-176.

SCHOOL OF MATHEMATICS, PHYSICS, COMPUTING AND ELECTRONICS

MACQUARIE UNIVERSITY

SYDNEY, NSW 2109

AUSTRALIA

Email: alan@mpce.mq.edu.au

DEPARTMENT OF MATHEMATICS

THE UNIVERSITY OF TEXAS AT AUSTIN

AUSTIN, TX 78712

USA

Email:nahmod@math.utexas.edu

CURRENT ADDRESSES:

HEAD CMA

AUSTRALIAN NATIONAL UNIVERSITY

CANBERRA ACT 0200

AUSTRALIA

Email: alan@wintermute.anu.edu.au

DEPARTMENT OF MATHEMATICS

LEDERLE GRT BOX 34515

UNIVERSITY OF MASSACHUSETTS

AMHERST, MA 01003

USA

Email: nahmod@math.umass.edu 